\title{
Exploring efficient seamless handover in VANET systems using network dwell time
}

\author{
Arindam Ghosh ${ }^{*}$, Vishnu Vardhan Paranthaman ${ }^{\dagger}$, Glenford Mapp ${ }^{\dagger}$ and Orhan Gemikonakli ${ }^{\dagger}$
}

\begin{abstract}
Vehicular ad hoc networks are a long-term solution contributing significantly towards intelligent transport systems (ITS) in providing access to critical life-safety applications and services. Although vehicular ad hoc networks are attracting greater commercial interest, current research has not adequately captured the real-world constraints in vehicular ad hoc network handover techniques. Therefore, in order to have the best practice for vehicular ad hoc network services, it is necessary to have seamless connectivity for optimal coverage and ideal channel utilisation. Due to the high velocity of vehicles and smaller coverage distances, there are serious challenges in providing seamless handover from one roadside unit (RSU) to another. Though other research efforts have looked at many issues in vehicular ad hoc networks (VANETs), very few research work have looked at handover issues. Most literature assume that handover does not take a significant time and does not affect the overall VANET operation. In our previous work, we started to investigate these issues. This journal provides a more comprehensive analysis involving the beacon frequency, the size of beacon and the velocity of the vehicle. We used some of the concepts of Y-Comm architecture such as network dwell time (NDT), time before handover $\left(T_{\mathrm{BH}}\right)$ and exit time (ET) to provide a framework to investigate handover issues. Further simulation studies were used to investigate the relation between beaconing, velocity and the network dwell time. Our results show that there is a need to understand the cumulative effect of beaconing in addition to the probability of successful reception as well as how these probability distributions are affected by the velocity of the vehicle. This provides more insight into how to support life critical applications using proactive handover techniques.
\end{abstract}

Keywords: IEEE802.11 p; Beaconing; Vehicle to infrastructure communication; Handover; Proactive handover; Network dwell time

\section{Introduction}

The rapid growth of the number of cars on the roads has created a plethora of challenges for road traffic management authorities, such as traffic congestion, increasing number of accidents, and air pollution. Over the last decade, significant research efforts from both automotive industry and academia have been underway to accelerate the deployment of a wireless network based on short-range communications among moving vehicles (vehicle-to-vehicle, V2V) and roadside infrastructure (vehicle-to-infrastructure, V2I). This network is called a vehicular ad hoc network (VANET) and is characterised by high node speed, rapidly changing topologies and short connection lifetimes.

\footnotetext{
*Correspondence: A.Ghosh@mdx.ac.uk

${ }^{\dagger}$ Equal contributors

Department of Computer and Communications Engineering, Middlesex University London, London, UK
}

Several applications for VANETs have been categorised for road-safety, traffic efficiency, and infotainment applications (i.e. information and entertainment applications). The latter two can be typically referred to as non-safety applications; they aim to provide information and comfort/entertainment to travellers and have the great potential to increase the chances of success for VANETs and to accelerate their market penetration [1]. Road traffic management for smart cities involves monitoring the actual traffic situation in real time (including volumes, speeds and incidents) and then controlling or influencing the flow using that information in order to reduce traffic congestion, deal efficiently with incidents and provide accurate and reliable traffic information and prediction to both drivers and authorities [2].

Beacons are used to discover and maintain neighbour relationships [3-5]. The European ITS VANET Protocol

\section{是 Springer}

(c) 2014 Ghosh et al.; licensee Springer. This is an Open Access article distributed under the terms of the Creative Commons Attribution License (http://creativecommons.org/licenses/by/2.0), which permits unrestricted use, distribution, and reproduction in any medium, provided the original work is properly credited. 
(EIVP) defines beacons as a cooperative awareness message (CAM) [6,7]. Beacons also include a security component, and the size of a beacon is approximately 400 bytes long [3-5]. Beaconing can be used for reliability due to the lack of acknowledgements and reservation by means of RTS/CTS [8]. Beacon messages are generated and issued periodically between the vehicle-to-vehicle (V2V) and vehicle to the roadside unit (RSU) i.e. (V2I) [6,7]. The generation rate is the rate at which beacons are sent to the MAC for transmission. Since they are used to create a cooperative awareness, beacon generation rate should be in the order of several beacons per second to provide the system with accurate information about the close surroundings $[3,5,8,9]$. Beacon frequency is the beacon generation rate which is denoted by $(\lambda)$. Though some research efforts consider a fixed $\lambda$ of $10 \mathrm{~Hz}$ [10], we motivate in [8] that generation rate adaptation as a network layer mechanism is one of the instruments to make beaconing more scalable. Increasing $\lambda$ results in more beacons being sent and a higher temporal resolution. But this comes at the price of an increase in collision probability, especially in dense traffic. Hence, an adaptive beaconing is preferable [5].

In the next couple of years, it is evident that intelligent transport systems (ITSs) will entail the deployment of VANETs especially in smart cities. For this purpose, it is imperative not only to have a proper infrastructure with several RSUs being placed in a resourceful and costeffective manner but also to serve the main purpose of ITS in order to have seamless connectivity for optimum coverage with ideal channel utilisation where vehicles are able to access applications and services quickly [2]. The paradox of deployment issues are that, on the one hand, ITSs demand the deployment of the infrastructure in such a way that it supports seamless connectivity, but on the other hand, this comes at the cost of having many RSUs placed along the roadside leading to interference issues. Hence, in order to achieve seamless connectivity, the placement of RSUs within the general infrastructure needs to be fully investigated [8].

Though other research efforts have looked at many issues in VANET networks, very few papers have looked at handover issues. Most papers assume that handover does not take a significant time and does not affect overall VANET operation. The Y-Comm architecture [11] was developed to explore proactive handover issues in future mobile networks. It has introduced an advanced handover classification system as well as new concepts such as network dwell time (NDT), time before handover $\left(T_{\mathrm{BH}}\right)$ and exit time (ET) [12]. In, our previous work [2], we used NDT, $T_{\mathrm{BH}}$ and ET [8] to analyse the handover issues in VANET systems. In this context, NDT is the time the vehicle spends in a RSU's coverage range. The results showed that the real-time NDT (NDTr) measured using a simulation is not equal to the theoretical NDT or ideal NDT (NDTi) which is calculated using Y-Comm techniques in [12]. The simulation was performed in OMNeT++ [13] using the Veins framework. The results clearly showed that the NDT was affected by frequency of beaconing as well as the velocity of the vehicle.

The aim of this journal article is to further investigate the effect of these parameters on NDTr and its ability to reach the NDTi, the ideal NDT with different beacon sizes and the different velocities of a vehicle. This was done by looking at entrance and exit scenarios using the Veins framework simulation focusing on MAC and PHY layers in analysing the factors contributing to NDTr. This study points to the need to develop a more comprehensive set of equations that can be used to calculate NDT in VANET systems and hence help us to understand the main handover issues in VANETs.

The contributions of this journal are as follows:

- To show the differences in the NDTi and NDTr based on the frequency and sizes of the beacon for two different velocities of a vehicle.

- To show the orthogonal relation between the frequency and size of the beacon.

- To investigate the relationship of cumulative probability distribution and its effect on measured NDT.

- To investigate how the probability distribution of successful beacon reception changes with velocity.

- To provide a step towards the modelling of a realistic NDT.

The rest of this journal article is structured as follows: Section 2 provides an overview of the related work in this area. Section 3 highlights on our previous work. Simulation background and setup are explained in Section 4. In Section 5, the simulation results and discussions have been critically evaluated. Section 6 demonstrates analysis which highlights the analytical approach towards the study. Finally, Sections 7 and 8 conclude this journal article and show the future work.

\section{Related work}

In [4], the author highlighted the importance of scalable beaconing and the fact that power control alone will not be sufficient if the requirement of the application has to be met. Hence, the rate at which beacons are generated must also be controlled. The author proposed an adaptive architecture and adaptive timing aspects of beacon generation.

In [14], the author proposed a distributed routing protocol and focuses on two kinds of handovers: inter RSU handover and intra RSU handover. The approximate location of the cars is found using the link quality based on 
received signal strength indicator (RSSI) from the timing advertisement from RSU.

In [15], a multi-technology seamless handover mechanism for vehicular networks is explored. The authors look at integrating other technologies like 3G to achieve seamless communication between the vehicle and the infrastructure without breaking an active session. Using extended mobility protocols of MIPv6 and PMIPv6, a test was performed to measure the handover latency for different bit rates between the same communication technology and between different communication technologies. Here, speeds of the vehicles considered were 50 and $60 \mathrm{Km} / \mathrm{h}$ respectively.

In [9], a time coordinated medium access control (MAC) protocol named WAVE point coordination function (WPCF) for vehicle to infrastructure (V2I) communication is investigated. The service disconnection time of various channel access techniques for V2I handover was shown. In order to reduce the handover delay and for a soft handover to happen, additional messages were added.

The work in [16], conducted a field experiment which extensively analysed the performance of V2I communication in an urban environment for an effective and reliable RSU deployment. The field testing was conducted in the city of Bologna with four key scenarios. Three urban scenarios and a highway scenario were considered. The communication performance was measured in terms of the packet delivery ratio (PDR) as a function of the distance of the onboard unit (OBU) to the communicating RSU. The author has presented the reliable connectivity range (RCR) and unreliable connectivity range (UCR) as the distance to the RSU up to which the experienced PDR is above 0.7 and below 0.1 , respectively. The study was performed for two transmission power levels, i.e. $10 \mathrm{dBm}$ and $20 \mathrm{dBm}$, and has shown that high transmission power levels can significantly increase the RCR and UCR distances. The study has also shown the effect of non-line-of-sight (NLOS), antenna heights, traffic and heavy vehicles. NLOS has shown a significant impact on the communication. The minimum and maximum reliable communication range distance was $400 \mathrm{~m}$ and $800 \mathrm{~m}$ (approx.), respectively.

In [17], the author has referred and criticised the works of [18-20] where a predictive or proactive handover approach is proposed for 802.11 networks. Here, the author has proposed a proactive polling mechanism where the information about the approaching vehicles are forwarded to the next RSU, where the vehicles become part of the communication schedule even before entering the next RSU's transmission range. The next RSU starts polling early enough to account for a $20 \%$ increase in average speed over the distance between the two access points. When a vehicle experiences a significant decrease in average speed or leaves the highway entirely, the RSU cannot continue sending out proactive polling messages indefinitely. Hence, a decrease in average speed of $20 \%$ is allowed. The polling by the next RSU stops after this threshold. A new RSU after this should go through the regular connection setup process.

The work in [21], proposed a seamless handover scheme based on proactive caching of data packets. Here, when an OBU is about to leave the coverage area of an RSU, the buffered packets will be forwarded to the entire candidate RSUs. The new RSU which is one of the candidates will transmit the buffered packets to the OBU and a message is sent to the rest of the candidate RSUs to discard the cached packets.

In [22], a new architecture called the MYHand architecture for providing extended information in next generation network (NGN) scenarios is detailed. By using the IEEE 802.21 protocol basic schema [23] and part of the Y-Comm architecture [24], MYHand improves the handover managed by mobile devices (user centric management). A scenario with three access providers and a mobile user walking through the avenue was simulated by using network simulator 2 (NS2).

The work in [12] proposes a proactive handover policy using a simple mathematical model. Proactive handover facilitates minimise disruption due to service degradation or packet loss during handover by signalling to the higher layers that a handover is about to happen. This work shows how the NDT and time before vertical handover (TBVH) are calculated in heterogeneous environments. TBVH is the time a mobile node has got to hit the circle for handover given the velocity and direction. The paper analysed various vertical handovers (WLAN-3G, 3GWLAN) in their work.

In [25], a seamless proactive vertical handover algorithm was proposed which took into account the users preferences, network conditions, velocity of the mobile station and application requirements for selecting a candidate network for handover which is stable. The proposed algorithm calculates the residence time in a candidate network which has already been proposed and highlighted in [12]. This shows the importance of NDT in achieving a proactive handover.

To the best of our knowledge, no work has considered the importance of lower layers (i.e. PHY and MAC) in achieving an effective proactive handover.

\section{Our previous work}

In our previous work [2], we presented the various challenges in providing a ubiquitous communication in VANET systems using roadside units. The concepts of YComm like NDT, time to handover $\left(T_{\mathrm{EH}}\right)$ and ET were introduced and have shown the importance of those concepts in achieving a seamless communication. In the context of VANET systems, NDT is the time the mobile unit 
spent under the coverage of a given RSU; $T_{\mathrm{EH}}$ is defined as the time the mobile unit takes to handover to the next RSU; ET is the time the mobile unit spends in an RSU before it must be handover to another RSU.

In Figure 1, the diagram demonstrates the following:

- Detection range is the region where both the vehicle's receiver sensitivity threshold and the SNIR are met for the payload. Vehicles within this range of the transmitting RSU are able to decode packets.

- Data exchange range is the region where the actual data transmission takes place.

- Time before handover is the region where the OBU gets ready for handover.

- Time to handover is the region where the actual handover takes place.

The work considered a very basic setup where there was no interference or other sources of noise, no effects of buildings and no traffic density issues in order to concentrate on the effect of beaconing and velocity of the vehicle on the network dwell time. Here, beacon size was kept constant at 656 bits.

Table 1 shows the NDT values from simulation experiments (i.e. NDTr) with different beacon generation frequencies $(\lambda)$ from the RSU and NDT using the formula in Equation 7 (i.e. NDTi) to calculate the upper bound. This upper bound does not consider any factors like contention, it assumes the medium or channel is ideal and that the only loss is due to propagation.

To investigate the minimum overlapping distance needed for a soft handover, simulation experiments were conducted for three different $\lambda \mathrm{s}$ from the RSU and with different velocities of the vehicle. In order to fully investigate handover issues, the two RSUs were moved closer until a fair amount of beacons from both RSUs simultaneously are received by the moving vehicle in the overlapping region. The results have been illustrated in Table 2.

The work concluded that a proactive handover approach is necessary for providing a ubiquitous communication in VANET systems and a realistic analytical model for NDT has to be developed in achieving this proactive handover. This shows that in order to provide seamless communication, NDT should be segmented as shown in Figure 1. Our previous work clearly highlighted that there is a relationship between beaconing frequency and the NDT which was not previously considered either in papers that looked beaconing or handover. In addition, this work investigates the effect of size of the beacon and velocity of the vehicle. In order to achieve this, there is a need to look at PHY and MAC mechanisms which were not considered in previous studies where predicting a residence time in a network or network dwell time is thought to be easy. In this journal, we further investigate and present various factors contributing to this $\mathrm{NDTr}$ which will be supporting the development of a realistic analytical model in the future.

\section{Simulation background and setup}

For the simulation experiments, the discrete event simulation environment $\mathrm{OMNeT}++$ [13] is used in conjunction with the Veins framework [26,27]. This is a mobility simulation framework for wireless and mobile networks. A beaconing model using IEEE $802.11 \mathrm{p}$ was implemented in Veins framework by [26]. All the PHY and MAC

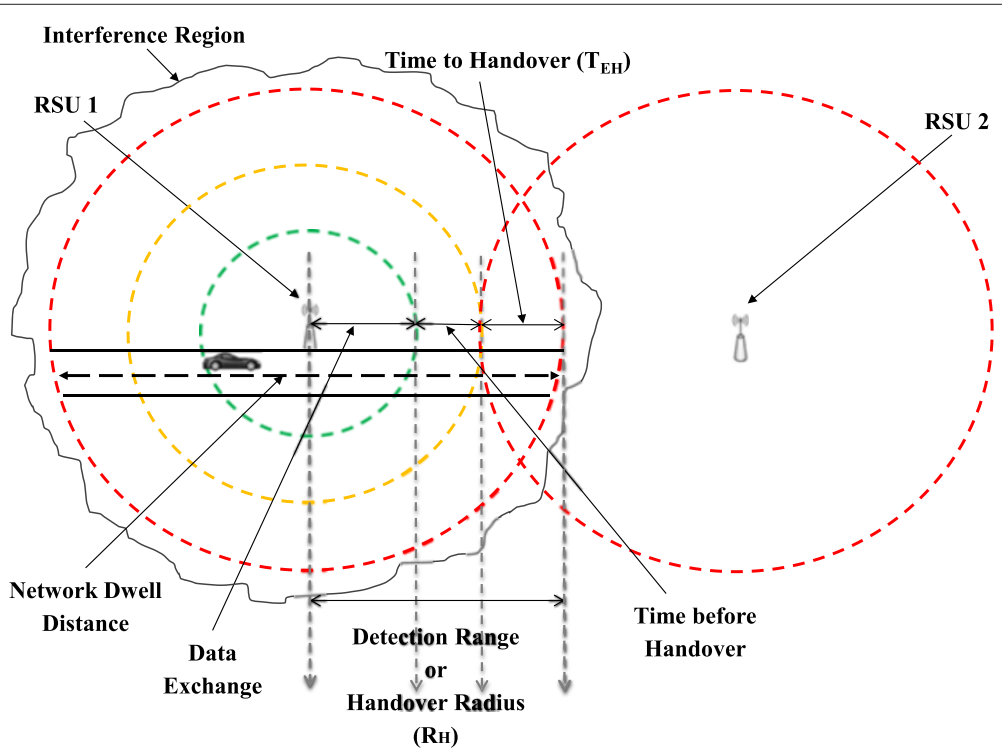

Figure 1 Transmitting RSU ranges. 
Table 1 Comparison of network dwell time from simulation with theoretical calculation

\begin{tabular}{|c|c|c|c|c|c|c|}
\hline \multirow[t]{2}{*}{ Speed } & \multirow[t]{2}{*}{ NDTi } & \multicolumn{5}{|c|}{ NDTr } \\
\hline & & $\lambda=1 \mathrm{~Hz}$ & $\lambda=5 \mathrm{~Hz}$ & $\lambda=10 \mathrm{~Hz}$ & $\lambda=20 \mathrm{~Hz}$ & $\lambda=40 \mathrm{~Hz}$ \\
\hline 0 to $108 \mathrm{~km} / \mathrm{h}$ & $60 \mathrm{~s}$ & $54 \mathrm{~s}$ & $55 \mathrm{~s}$ & $57 \mathrm{~s}$ & $57 s$ & $57 \mathrm{~s}$ \\
\hline $144 \mathrm{~km} / \mathrm{h}$ & $45 \mathrm{~s}$ & $37 s$ & $39 s$ & $43 \mathrm{~s}$ & $43 \mathrm{~s}$ & $43 \mathrm{~s}$ \\
\hline $180 \mathrm{~km} / \mathrm{h}$ & $36 s$ & $30 \mathrm{~s}$ & $31 \mathrm{~s}$ & $34 s$ & $34 \mathrm{~s}$ & $34 s$ \\
\hline
\end{tabular}

properties used in the IEEE 802.11p simulation model conform to $[28,29]$.

\subsection{Simulation scenario}

A stationary node (i.e. RSU) is placed as shown in Figure 2. Another mobile node (i.e. vehicle) is made to run over the range of the RSU for collecting various values for our study with two different velocities $(10 \mathrm{~m} / \mathrm{s}$ and $30 \mathrm{~m} / \mathrm{s})$. To understand and model a concept like NDT which no other work has ever considered, we first have to start with a simple scenario. We have considered a very basic setup where there are no interferences, no effects of buildings or any traffic density. Hence concentrating on the effects of beaconing, size of beacons and velocity of the vehicle on NDT. This will allow us to understand the key factors before studying more complex scenarios. A scenario as similar to Figure 2 is created.

During simulation, the RSU broadcasts the beacon with different $\lambda$ and with different beacon sizes for two different velocities. An overview of the different parameter values used is shown in Tables 3 and 4 . Note that these parameters contain the EDCA default values [6-8]. The remaining parameters are set according to the default values used by the Veins framework [26].

Beacon sizes of 100, 300, 500 and 723 bytes have been used in [30] for $6 \mathrm{Mbps}$ packet error ratio experiment. This result was used in the development of Veins framework in $6 \mathrm{Mbps}$ packet error rate modelling. Beacon size of 1,574 bytes was also used in an experimental study [31]. Further, this result was used in the development of Veins framework in $18 \mathrm{Mbps}$ packet error rate modelling. Hence, we have considered these sizes of beacon to conduct our study.

\subsection{Calculation of reception power}

The reception power is the power at which cars will receive the beacons, but this is dependent on the power

Table 2 Minimum overlapping needed for a soft handover

\begin{tabular}{lccc}
\hline Speed & \multicolumn{3}{c}{ Minimum overlapping needed } \\
\cline { 2 - 4 } & $\boldsymbol{\lambda}=\mathbf{1} \mathbf{~ H z}$ & $\boldsymbol{\lambda}=\mathbf{5 ~ H z}$ & $\boldsymbol{\lambda}=\mathbf{1 0 ~} \mathbf{H z}$ \\
\hline 0 to $108 \mathrm{~km} / \mathrm{h}$ & $415 \mathrm{~m}$ & $215 \mathrm{~m}$ & $136 \mathrm{~m}$ \\
$144 \mathrm{~km} / \mathrm{h}$ & $445 \mathrm{~m}$ & $285 \mathrm{~m}$ & $186 \mathrm{~m}$ \\
$180 \mathrm{~km} / \mathrm{h}$ & $525 \mathrm{~m}$ & $315 \mathrm{~m}$ & $206 \mathrm{~m}$ \\
\hline
\end{tabular}

at which the signal is transmitted. With a low transmission power, only the closest neighbour may receive the beacon, a more remote node might not. With a high transmission power, a significant number of cars might receive the beacon, but the collision probability is also higher [3] and more cars will receive interference. The goal of transmission power control is to increase spatial frequency reuse. The power control method must be fair: a higher transmission power of a sender should not be selected at the expense of preventing other vehicles to send/receive their beacons. In $[3,8]$, adaptive solutions to transmission power control are explored in detail. The minimum received power is calculated in the $\mathrm{OMNeT}++$ simulation module named Connection Manager [13]. The minimum power level at the car to be able to physically receive a signal from the RSU is shown below:

$$
\text { minRecvPow }=10^{\text {sat } / 10},
$$

where sat $\rightarrow$ minimum signal attenuation threshold.

\subsection{Calculation of detection range}

Calculation of the detection range (DR) $[13,26]$ based on transmitter power, wavelength, path loss coefficient and a

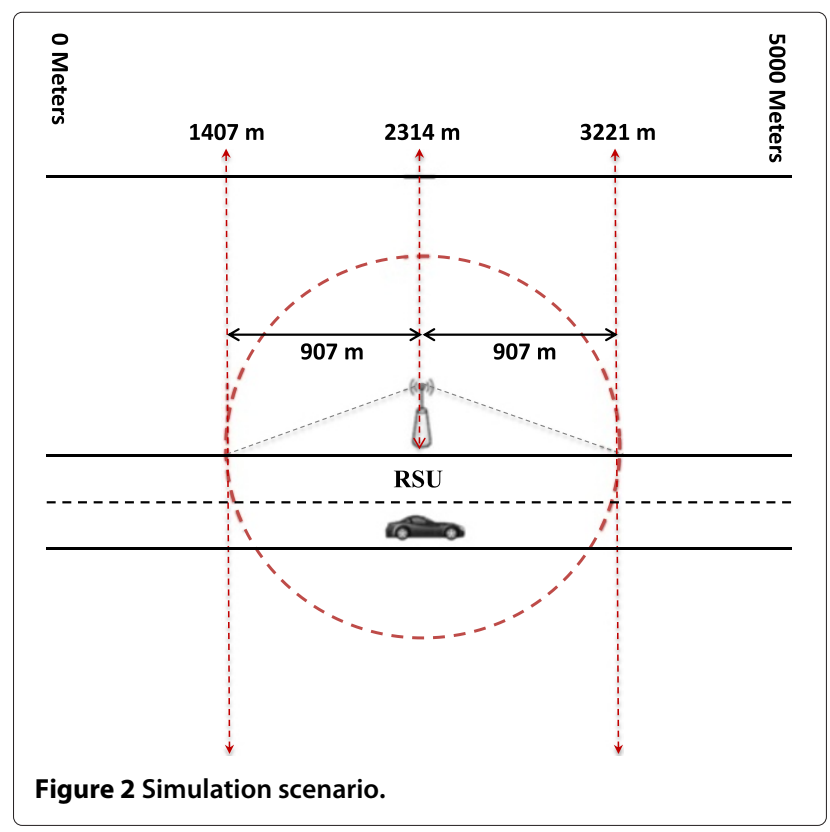


Table 3 RSU configuration parameters

\begin{tabular}{lc}
\hline Parameter & Values \\
\hline Transmission power & $20 \mathrm{~mW}$ \\
Bit rate & $18 \mathrm{Mbps}$ \\
Sensitivity & $-94.0 \mathrm{dBm}$ \\
Thermal noise & $-110.0 \mathrm{dBm}$ \\
Header length & 11 bytes \\
Beacon length & $100,300,500,723,1,574$ bytes \\
Send data & False \\
\hline
\end{tabular}

threshold for minimal received power for a communication to take place is shown below.

$$
D R=\left(\left(\Lambda^{2} p \text { Max }\right) /\left(16.0 \pi^{2} \text { minRecvPow }\right)\right)^{1 / \alpha},
$$

where $\Lambda \rightarrow$ wavelength $=$ (speedoflight $/$ carrierfrequency), $p$ Max $\rightarrow$ maximum transmission power possible, $\alpha \rightarrow$ minimum path loss coefficient, sat $\rightarrow$ minimum signal attenuation threshold and minRecvPow $\rightarrow$ minimum power level to be able to physically receive a signal.

Based on the simulation parameters as shown in Tables 3 and 4, the detection range is calculated in the simulation. The outcome from the formula suggests 907.84256 metres, i.e. the radius (R) of the coverage. For this reason, all the mathematical calculations in our work has considered 908 metres (approx.) as the radius of the coverage.

The simulation parameters with which the experiments were carried out are shown in the Tables 3 and 4.

\subsection{Calculation of successful packet reception in simulation}

In Figure 3, T1 and T2 is the time when the first packet at PHY and MAC layers are received, respectively. Between T3 and T4 is the region where the packet is always successfully received, i.e. where probability $(\mathrm{P})$ of successful packet reception is ' 1 '. T5 and T6 is the time when the last packet at MAC and PHY layers are received. All the packets between $\mathrm{T} 1$ to $\mathrm{T} 2$ and $\mathrm{T} 5$ to $\mathrm{T} 6$ are lost due to bit errors. Figure 3 also shows that the reliable communication starts only when the packet reaches the MAC layer. The reason and the way the packets are dropped by the simulation in the PHY layer is explained below.

\section{Table 4 OBU configuration parameters}

\begin{tabular}{lc}
\hline Parameter & Values \\
\hline Speed & $10 \mathrm{~m} / \mathrm{s}, 30 \mathrm{~m} / \mathrm{s}$ \\
& $(36 \mathrm{~km} / \mathrm{h}),(108 \mathrm{~km} / \mathrm{h})$ \\
Channel bandwidth & $10 \mathrm{MHz}$ \\
OBU receiver sensitivity & $-94.0 \mathrm{dBm}$ \\
\hline
\end{tabular}

In the simulation, $\mathrm{T} 2$ is the time where the actual communication starts and we know that we receive packet at $\mathrm{T} 1$, but these received packets are discarded due to bit errors; hence, the question which has to be asked here is, can this time $\mathrm{T} 2$ be determined given that the vehicle receives the first packet in PHY layer at time T1? To analyse this effect, we further carefully investigate in the calculation of the successful packet reception. Figure 3 shows that the communication starts only when the packet reaches the MAC layer.

The graph, shown in Figure 4, has been simulated in $\mathrm{OMNeT}++$ using the Veins framework. The graph shows the PacketOk and random double number. This simulation is carried on with one RSU and one vehicle moving at $30 \mathrm{~m} / \mathrm{s}$ as shown in the scenario. The beacon with a size of 100 bytes with a beacon generation frequency of $1 \mathrm{~Hz}$ is broadcasted by the RSU.

For each beacon received at the PHY layer, a PacketOk number is computed which is a packet reception ratio. This number is computed based on bit error rate (BER) and length of beacon. This computed double number is compared against a randomly generated double number ranging between 0 and 1 . If the computed number is less than the randomly generated number, then that respective beacon is dropped at the PHY layer, reason assumed that there is an error in the packet.

The lower (red) line is the randomly generated double number and the upper (blue) line is the computed PacketOk. The PacketOk number below the randomly generated number curve is assumed as error and has been dropped at the PHY layer. Packet delivery ratio in Veins for $18 \mathrm{Mbps}$ bit rate is calculated using the below formula which has been modelled using [31].

$$
\mathrm{PDR} \Rightarrow[1-1.5 \operatorname{erfc}(0.45 \sqrt{\mathrm{SNR}})]^{L},
$$

where

$$
\text { Signal to noise ratio }(\mathrm{SNR})=10^{\mathrm{SNR}_{d B} / 10}
$$

and $\mathrm{L} \rightarrow$ length of the packet.

In Figure 4, from the graph, we can observe that as the vehicle is heading towards the RSU, the packet reception probability increases and at a point reaches 1 which means there is no possibility of error in the packet. In other words, we can say that the region where the $P=1$ is a very reliable communication region. This is the time from $\mathrm{T} 3$ to $\mathrm{T} 4$ which has been shown in the Figure 3.

\section{Simulation results and discussion 5.1 Why NDT?}

NDT is the time a vehicle spends in a RSU's network range. If this time can be estimated even before a vehicle enters a network range, then the resources can be used in 


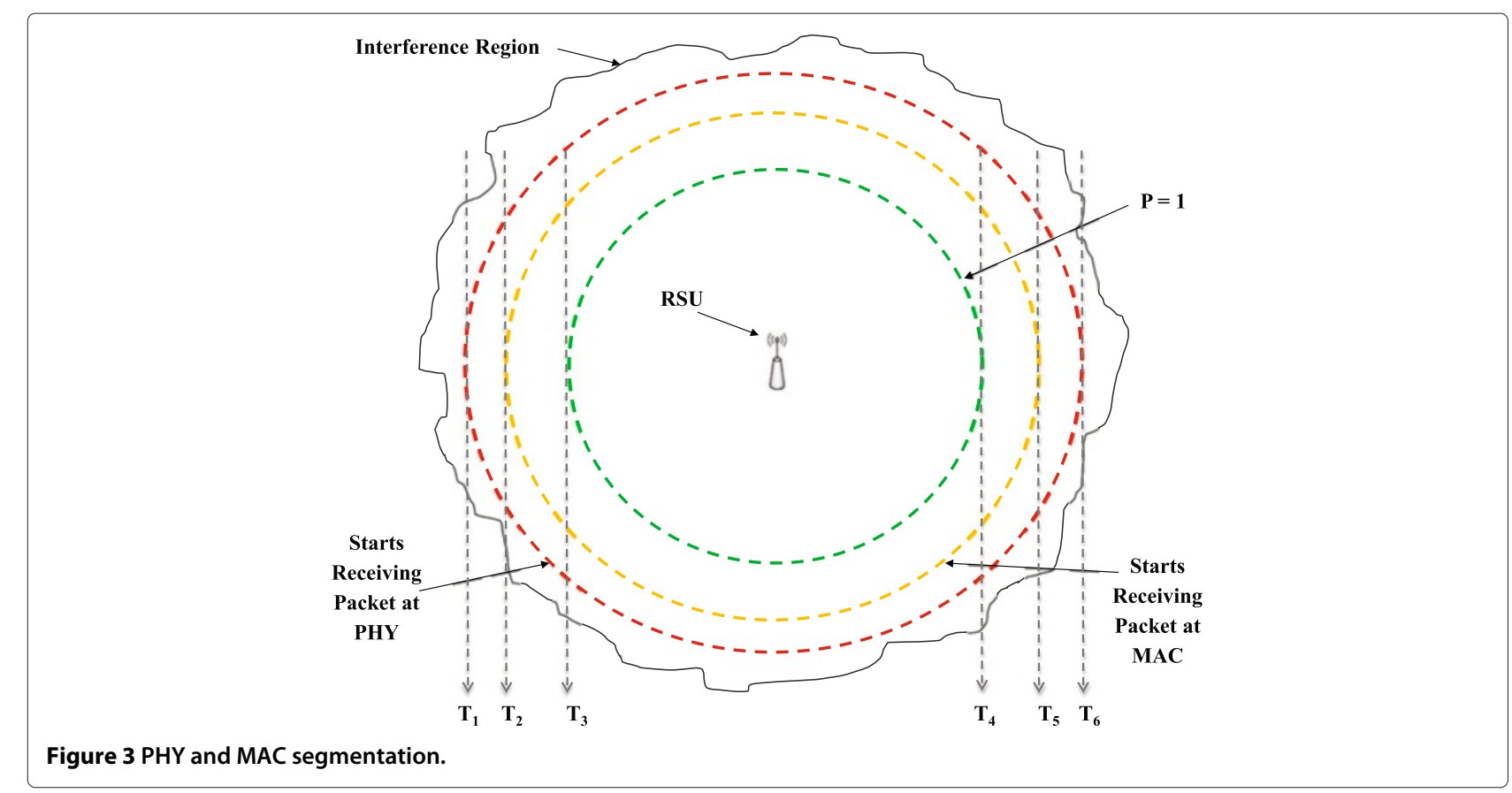

an efficient way and proactive handover can be made possible which ensures a ubiquitous communication. NDT in a wireless network is given by the reciprocal of the mobility leave rate. In the literature [12], the mobility leave rate is given by

$$
\mu_{m l}=E_{\mathrm{vel}} \times P /(\pi \times A),
$$

where $E_{\mathrm{vel}} \rightarrow$ expected velocity of the car, $\mathrm{P} \rightarrow$ perimeter of the cell and $\mathrm{A} \rightarrow$ area of the cell.

$$
\mathrm{NDT}=1 / \mu_{m l}=\left(\pi \times R_{H}\right) / V_{\max },
$$

where $\mu_{m l} \rightarrow$ mobility leave rate from Equation $5, R_{H} \rightarrow$ handover radius and $V_{\max } \rightarrow$ maximum velocity of the vehicle

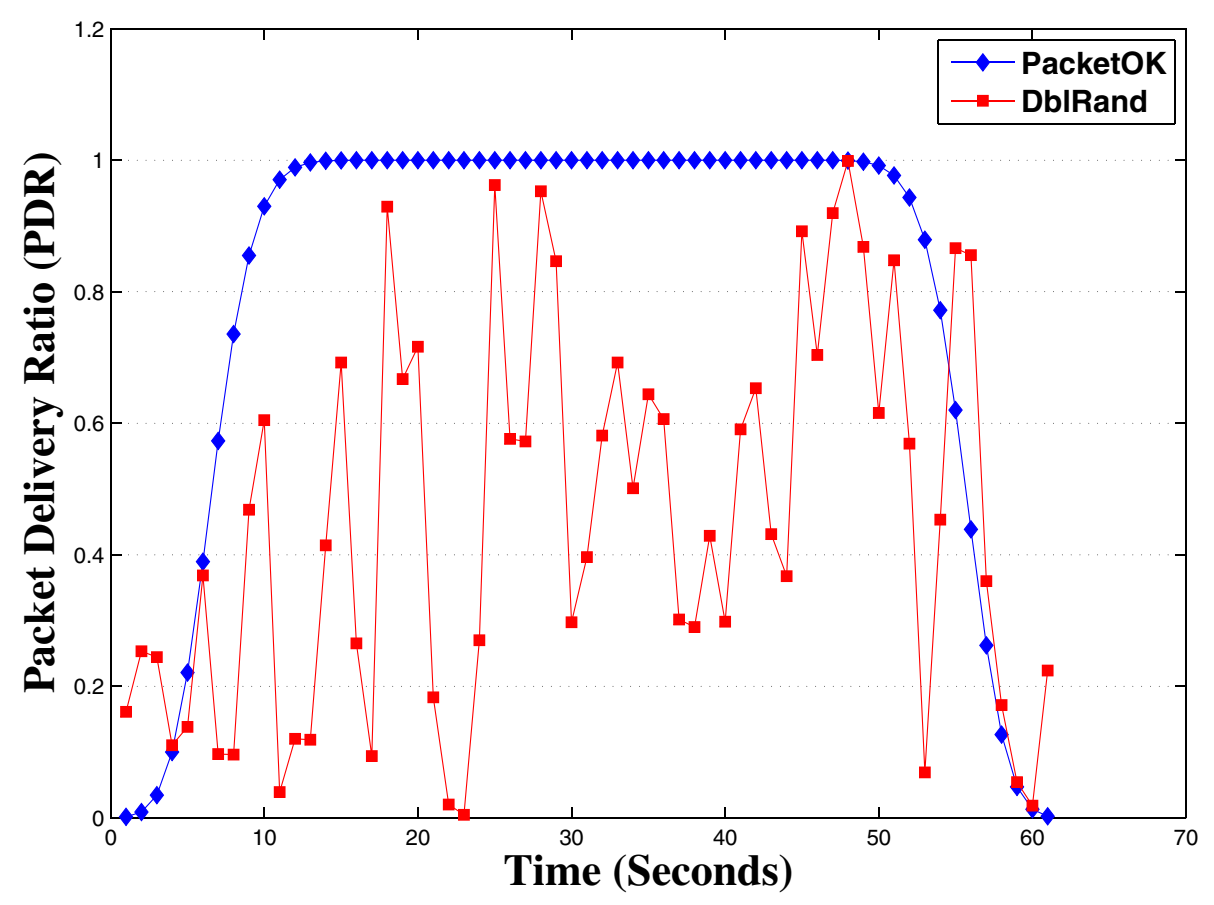

Figure 4 PacketOK vs DbIRand. 
In a motorway context, the distance between two travelling points can be directly calculated. Hence, NDT is given as shown below.

$$
\mathrm{NDT}=\mathrm{NDD} / E_{\mathrm{vel}},
$$

where NDD is network dwell distance travelled along a motorway that is in coverage of a given network [12]. The exact distance between two points on a motorway can be calculated using GPS. For our study in VANETs, we assume that the RSU is alongside a straight road; hence, NDD is approximately equal to $2 \mathrm{R}$ where $\mathrm{R}$ is the radius of coverage Figure 5.

With ideal NDT denoted as (NDTi), it is assumed that the communication starts as soon as the vehicle hits the edge of the coverage of a communication range. However, in real time, the measured definition of NDT, NDTr, can be defined as the time between the first and the last beacon reaching the MAC layer without being dropped in the PHY layer due to bit error as shown in Figure 6.

The graph in Figure 7 shows the NDTr for different size of beacon broadcasted to the vehicle moving at a constant speed $(10 \mathrm{~m} / \mathrm{s})$ with different $\lambda$. Figure 8 shows the same but with a different speed $(30 \mathrm{~m} / \mathrm{s})$. The NDTi is also plotted in this graph. It shows that as the size of the beacon increases, the NDTr is reduced, i.e. the communication time is reduced. NDT also reduces (comparing Figures 7 and 8 ) as the velocity of the vehicle increases. This clearly shows that the size of the packet is an important factor in determining the NDTr. The graphs also clearly show that there is no peak increase in NDTr after $10 \mathrm{~Hz}$ and it is also evident that some beacons are being dropped which causes this difference between NDTr and NDTi.
The reason and the way the beacons are dropped by the simulation in the PHY layer is explained below.

\subsection{Further investigation into PHY layer in relation to beacon size}

Hence, for further investigation, simulation was carried out but this time monitoring the beacons received at the lower layer (i.e. PHY). The graphs in Figures 9 and 10 show the first beacon reception at both PHY and MAC layers against simulation time during the entry by the vehicle in the coverage region. The simulation was carried out for different sizes of beacon with different beacon frequencies with two different velocities of the vehicle. It also shows the actual interference range or detection range calculated in the Veins framework comparing with the actual time the beacon was received.

This clearly showed that the vehicle starts receiving the beacon at the PHY layer as soon as it enters the detection range. This detection range is the place where the minimum criteria for the communication to happen are met. The time delay between the PHY layer first beacon and the MAC layer first beacon is due to the loss of those beacons, i.e. those beacons are received by the PHY layer but with errors in the beacon and hence dropped at the PHY layers as depicted in Figure 11. We can also see that when there is an increase in size of the beacon, there is a delay in reception of the beacon at the MAC layer, i.e more beacons are lost due to error at the PHY layer. When comparing the graphs in Figures 9 and 10 with the graphs in Figures 7 and 8 , respectively, we can conclude that the increase in size of the beacon will push the NDT down.

Figures 12 and 13 show the last beacon reception at both $\mathrm{PHY}$ and MAC layers against simulation time during the exit by the vehicle from the coverage region.

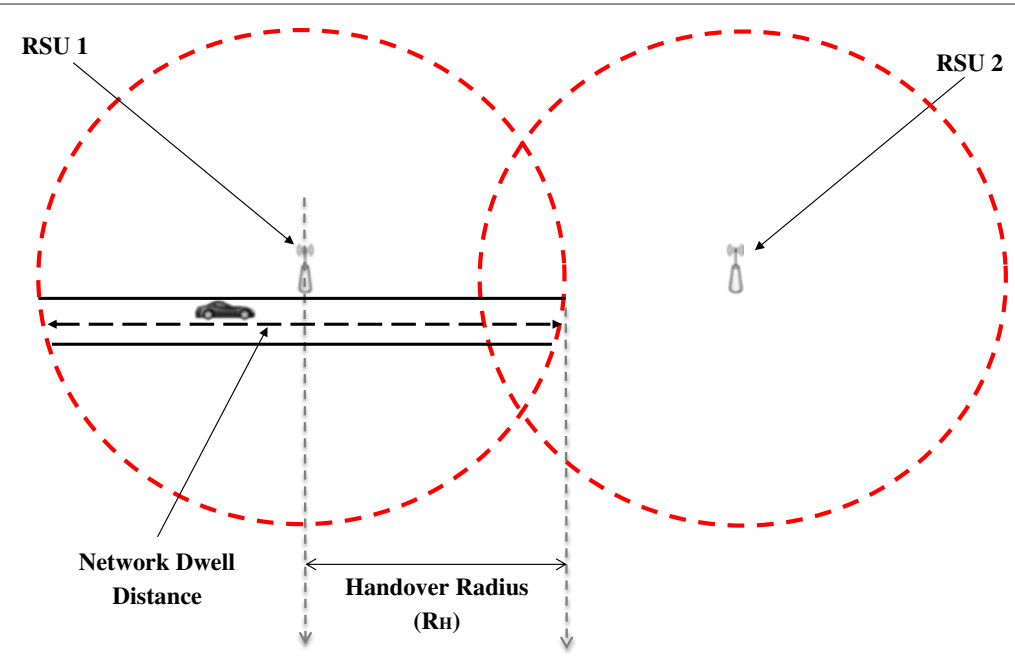

Figure 5 Handover radius. 


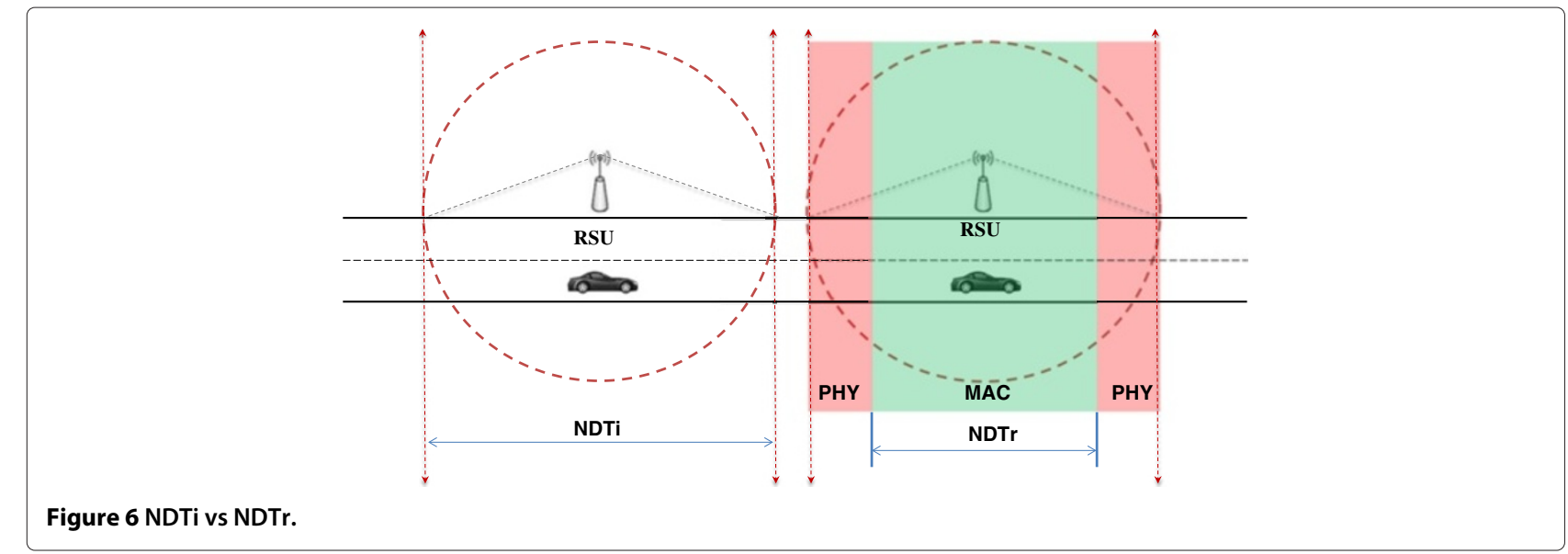

\section{Analysis}

\subsection{Cumulative probability calculations}

In order to investigate the effect of beacon frequency, we also need to look at the cumulative probability of a successful packet reception, in addition to calculating the probability of a successful packet reception for an individual packet at a given time ' $t$ '. Since we know the single packet reception probability using Equation 8 from the simulation, the cumulative probability can be calculated.

Therefore, if $P$ is the probability of a successful reception, then the cumulative probability for a sequence of $N$ receptions is given by:

$$
P+(1-P) P+(1-P)^{2} P+\ldots \ldots+(1-P)^{N-1} P .
$$

In probability theory, $P$ is constant and cumulative probability (CP) tends to 1 as $N$ tends to infinity. In this case, it means that successful reception of the beacon is guaranteed once the CP reaches 1 . But in this scenario because the vehicle is moving towards the RSU, $P$ increases for every sequence. Therefore, for $N$ receptions, the CP is

$$
\mathrm{CP}=P_{1}+\left(1-P_{1}\right) P_{2}+\left(1-P_{1}\right)\left(1-P_{2}\right) P_{3}+\ldots,
$$

where, $P_{N}$ is greater than $P_{N-1} \ldots=1$.

Since $P$ is increasing because the vehicle is moving towards the RSU, hence the cumulative probability reaches ' 1 ' long before infinity and therefore affects the

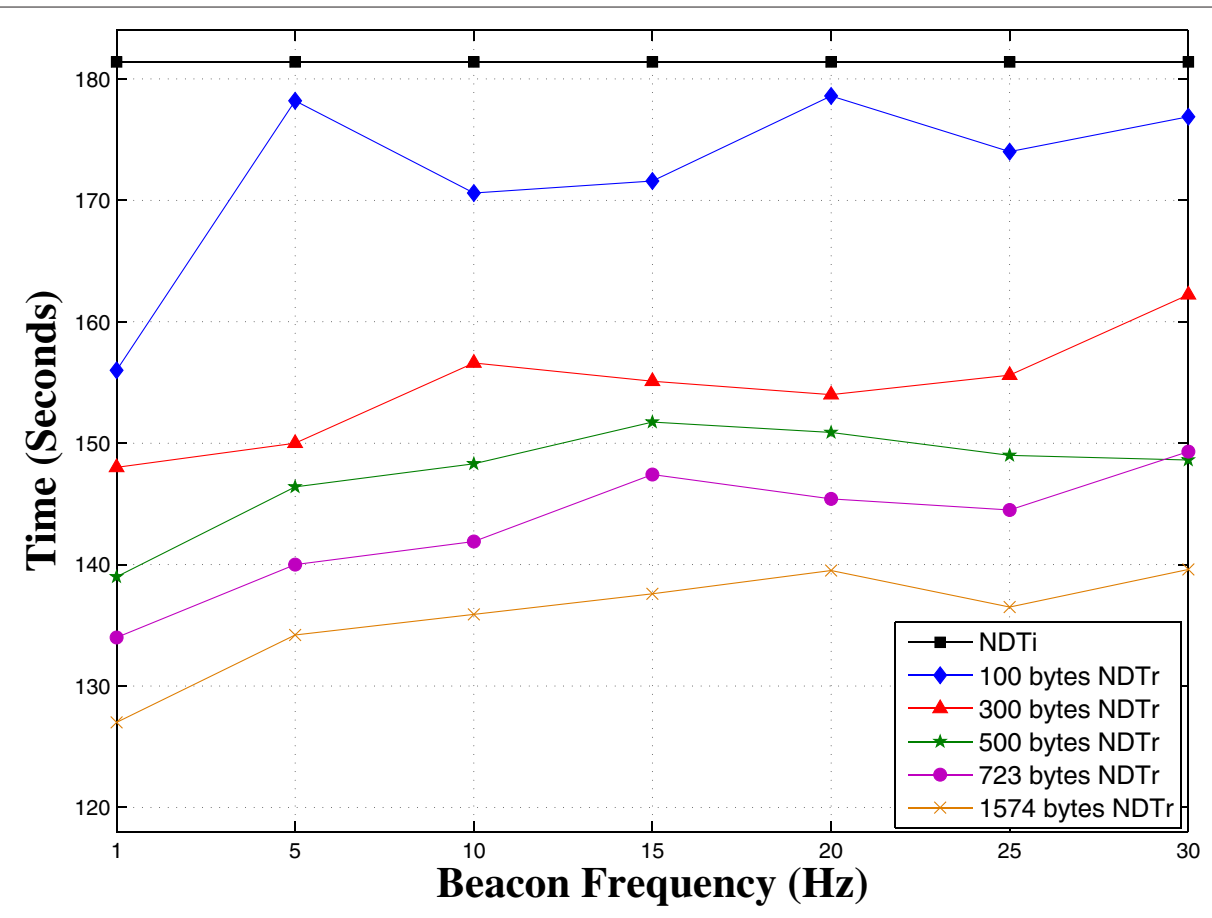

Figure 7 NDTr with different beacon sizes $(10 \mathrm{~m} / \mathrm{s})$. 


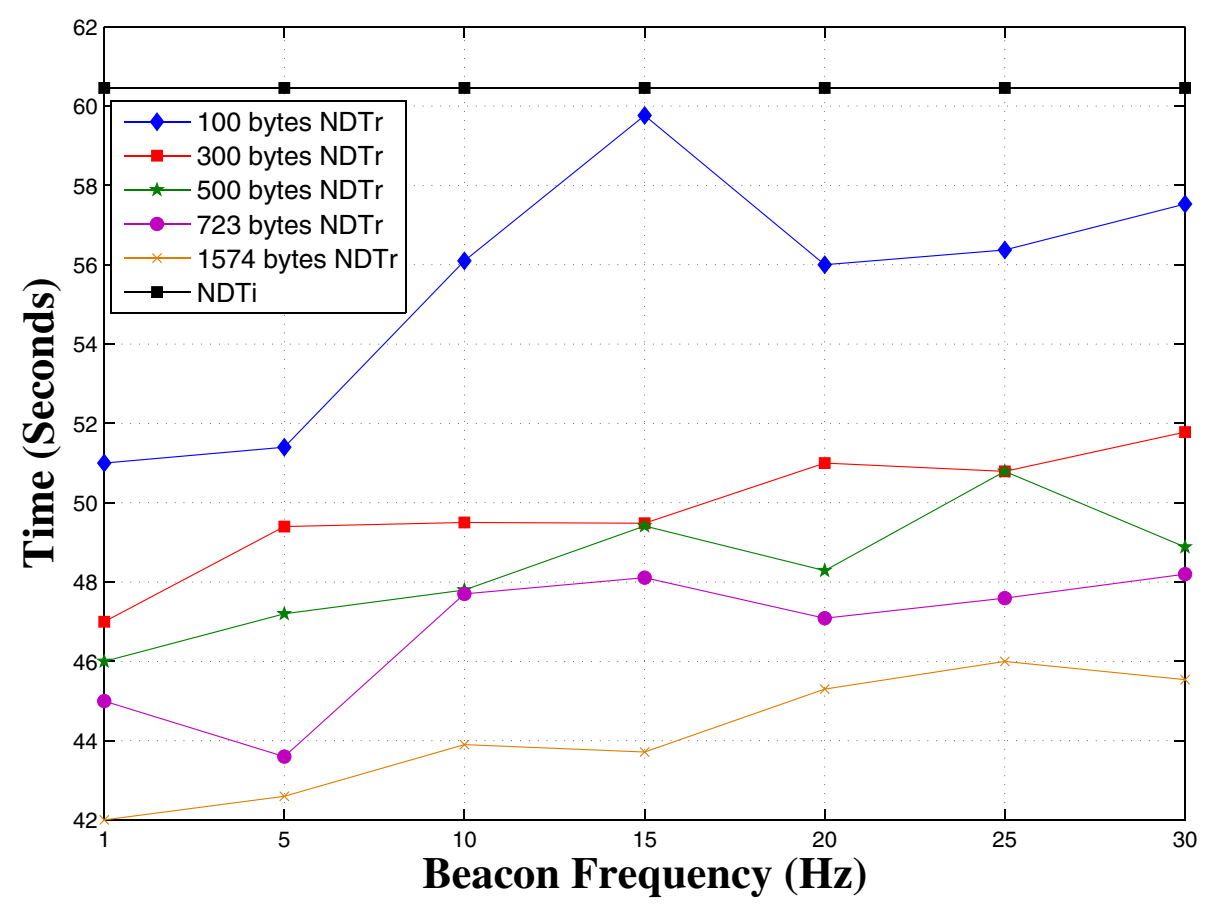

Figure 8 NDTr with different beacon sizes $(30 \mathrm{~m} / \mathrm{s})$.

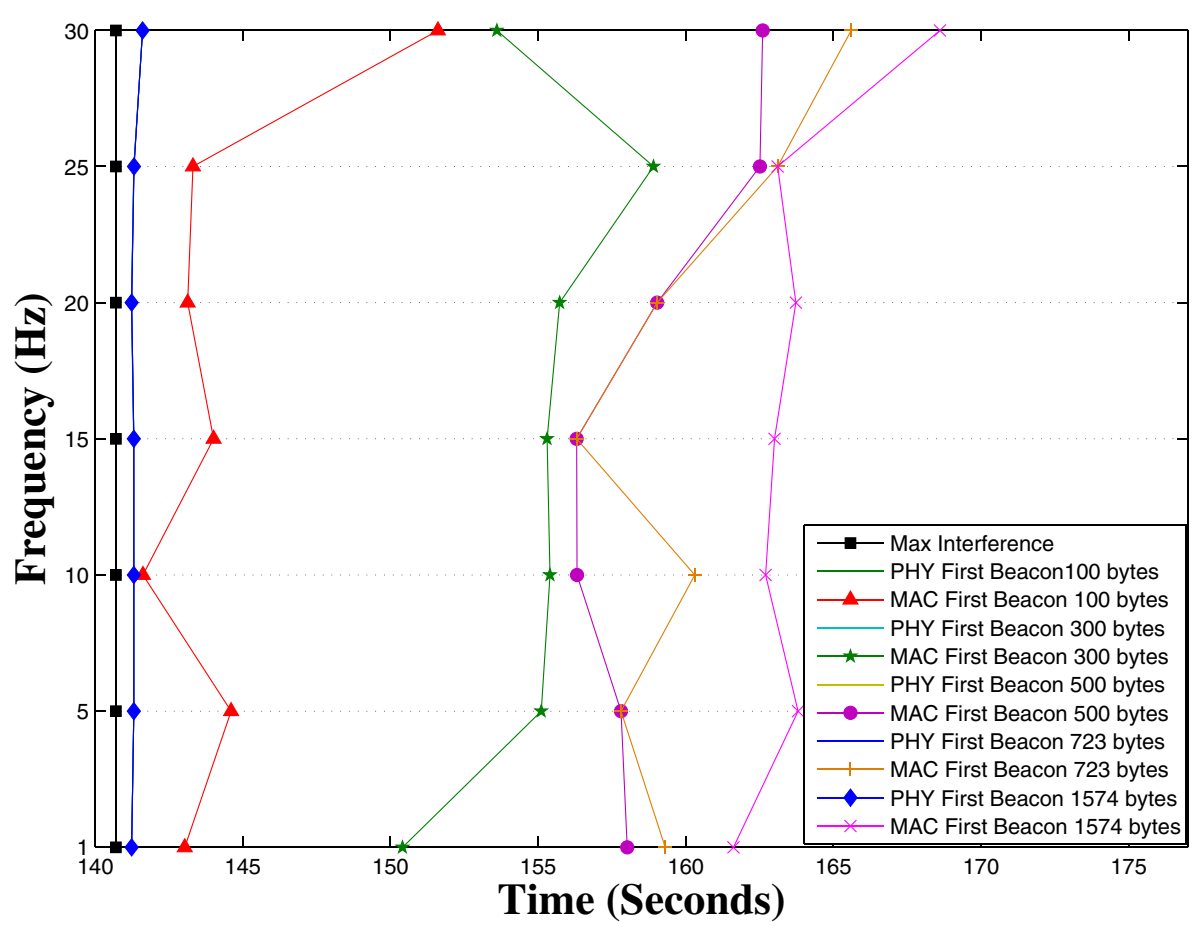

Figure 9 Entry side of coverage area $(10 \mathrm{~m} / \mathrm{s})$. 


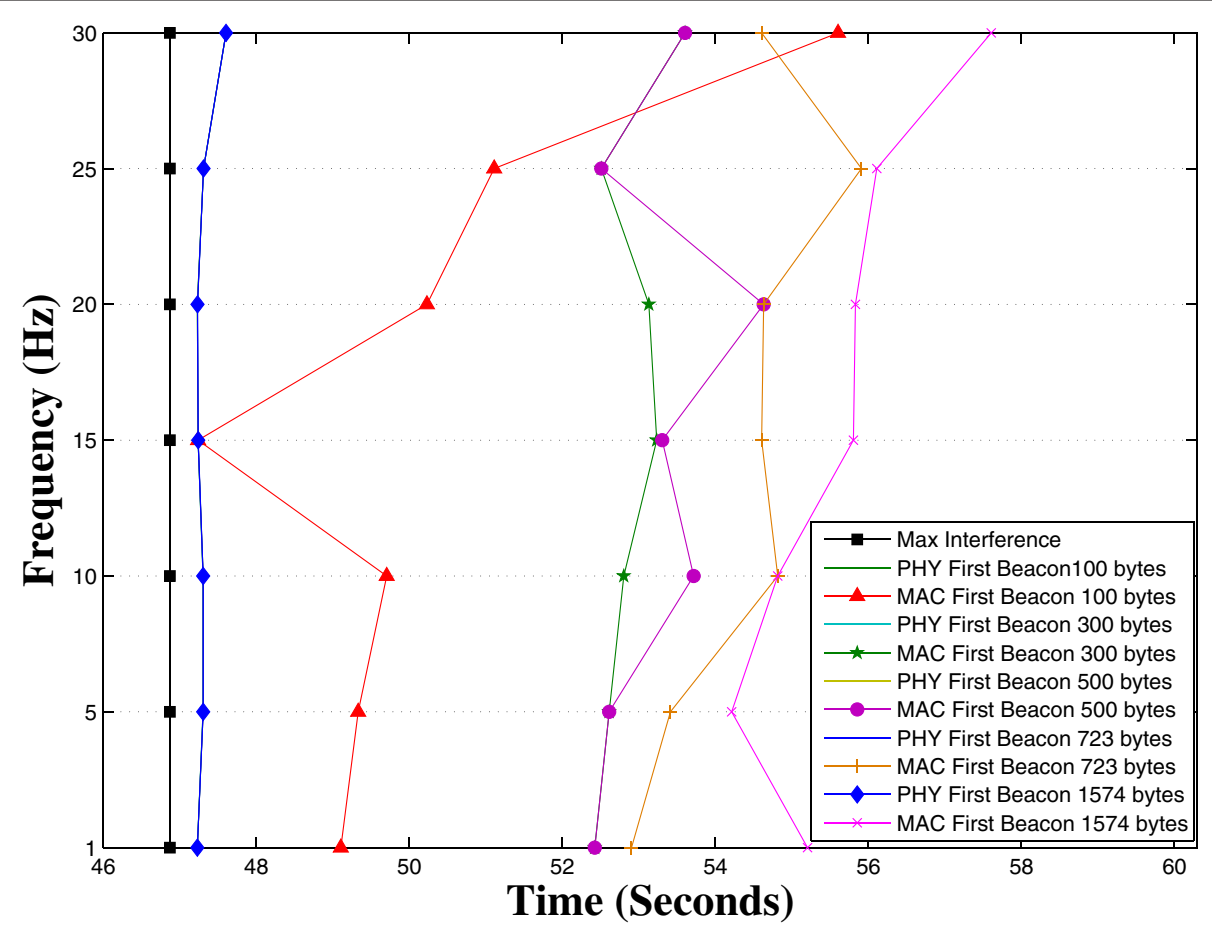

Figure 10 Entry side of coverage area $(30 \mathrm{~m} / \mathrm{s})$.

successful reception of the beacon. This analysis applies when the vehicle enters the network.

For exit times, we consider the probability of not receiving the packet $P_{n}=1-P$ from the RSU as we drive away, i.e., the negative cumulative probability. If $P$ is the probability of successful reception the negative cumulative probability $\left(\mathrm{CP}_{n}\right)$ is given by:

$\mathrm{CP}_{n}=\left(1-P_{1}\right)+P 1\left(1-P_{2}\right)+P 1 . P 2\left(1-P_{3}\right)+\ldots$.

For the exit scenario $P$ the probability of the successful reception decreases as we move away from the

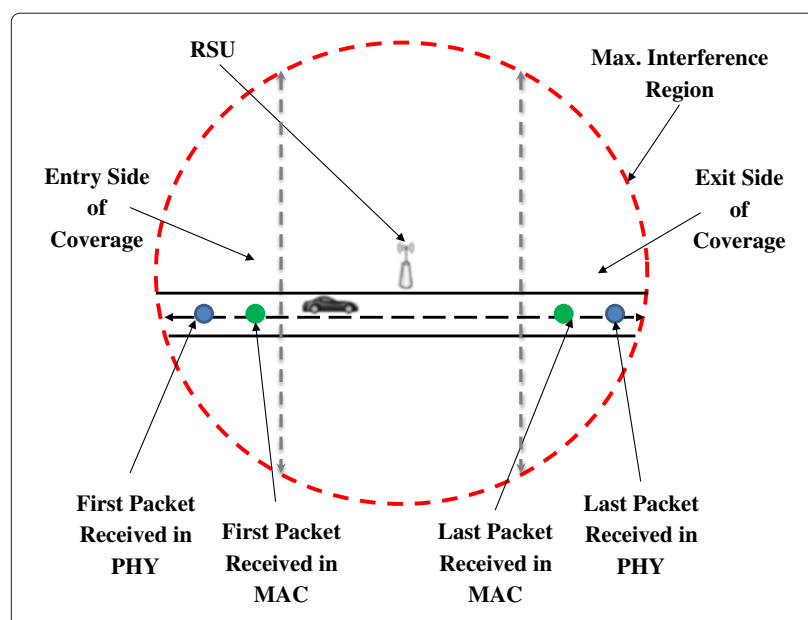

Figure 11 First and last beacon received at PHY and MAC layers.
RSU; hence, $1-\mathrm{P}$ is increasing. Once the vehicle does not hear the beacon after the period $T$, the inverse of the beacon frequency, it immediately hands over to the next RSU. Our results consider the effect of the cumulative probability on entrance and exit regions of RSU coverage.

\subsection{Cumulative probability effect on beacon frequency}

To understand the effect of frequency in determining the NDTr, the CP of the packet reception rate reaching ' 1 ' (i.e. vehicle moving towards RSU) has been calculated for different frequency and different sizes of beacon for the entry region. The $\mathrm{CP}$ of this entry region for two different velocities $(10 \mathrm{~m} / \mathrm{s}$ and $30 \mathrm{~m} / \mathrm{s})$ of a vehicle is shown in Figures 14 and 15, respectively. The result presented as graphs in Figures 14 and 15 show that the cumulative probability reaches ' 1 ' long before the probability of an individual successful beacon reception, and therefore, this parameter better explains the relationship between beacon frequency and successful reception and not the individual probability.

The graph in Figure 15 shows that as the frequency increases, the CP is reaching ' 1 ' much before the actual probability. Here, after $10 \mathrm{~Hz}$ to $15 \mathrm{~Hz}$, there is not much decrease in the time. This shows the impact of the frequency on the NDT. The first result shows that the cumulative probability reaches ' 1 ' long before the probability of an individual successful reception, and therefore, it is the 


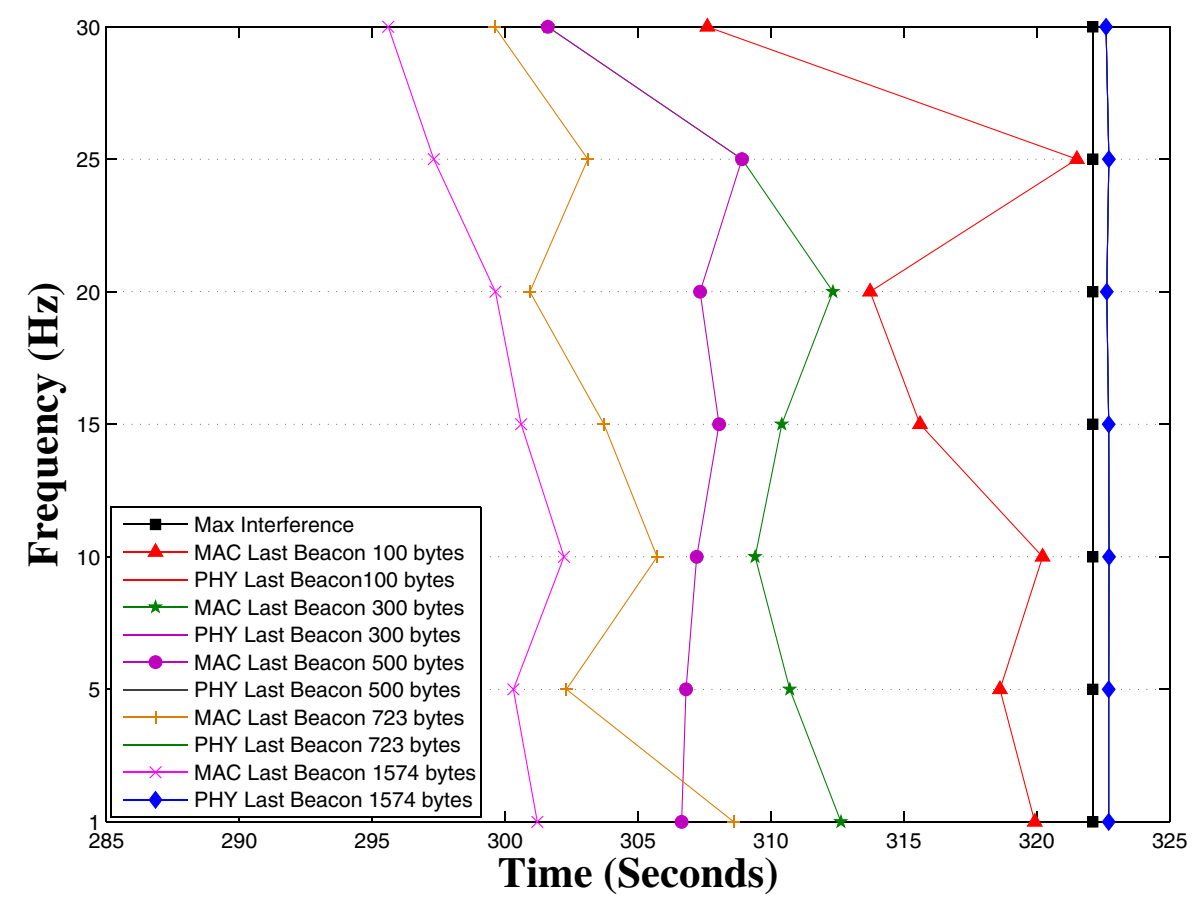

Figure 12 Exit side of coverage area $(10 \mathrm{~m} / \mathrm{s})$.

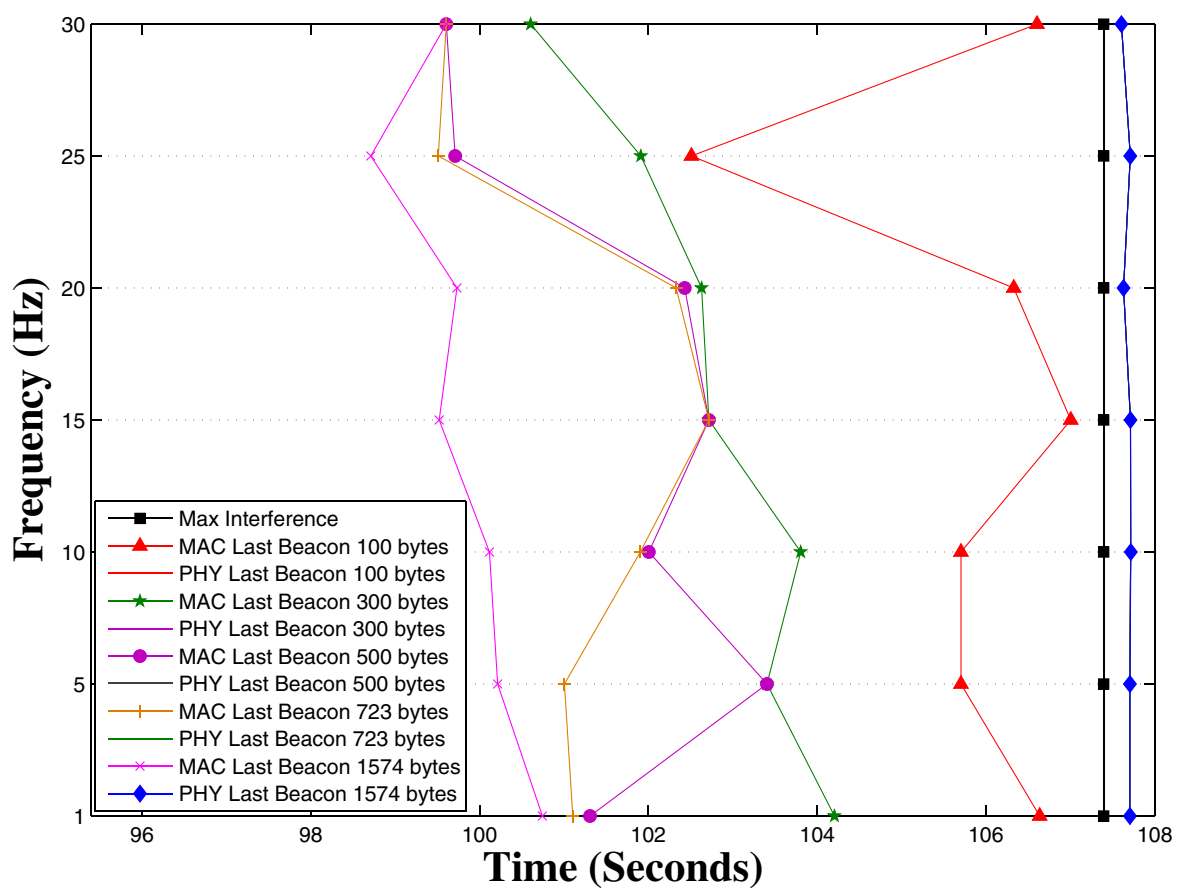

Figure 13 Exit side of coverage area $(30 \mathrm{~m} / \mathrm{s})$. 


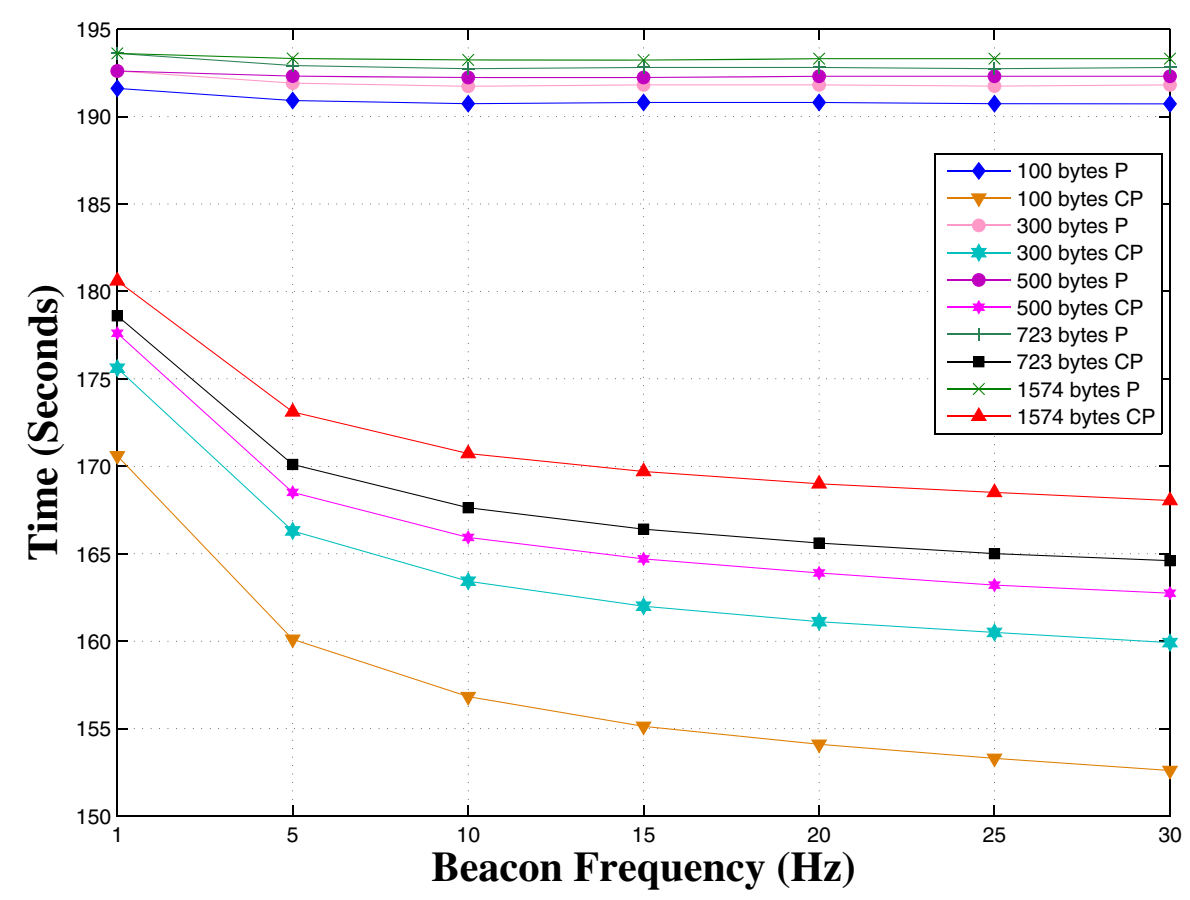

Figure $14 \mathrm{CP}$ reaching 1 (entry region $-10 \mathrm{~m} / \mathrm{s}$ ).

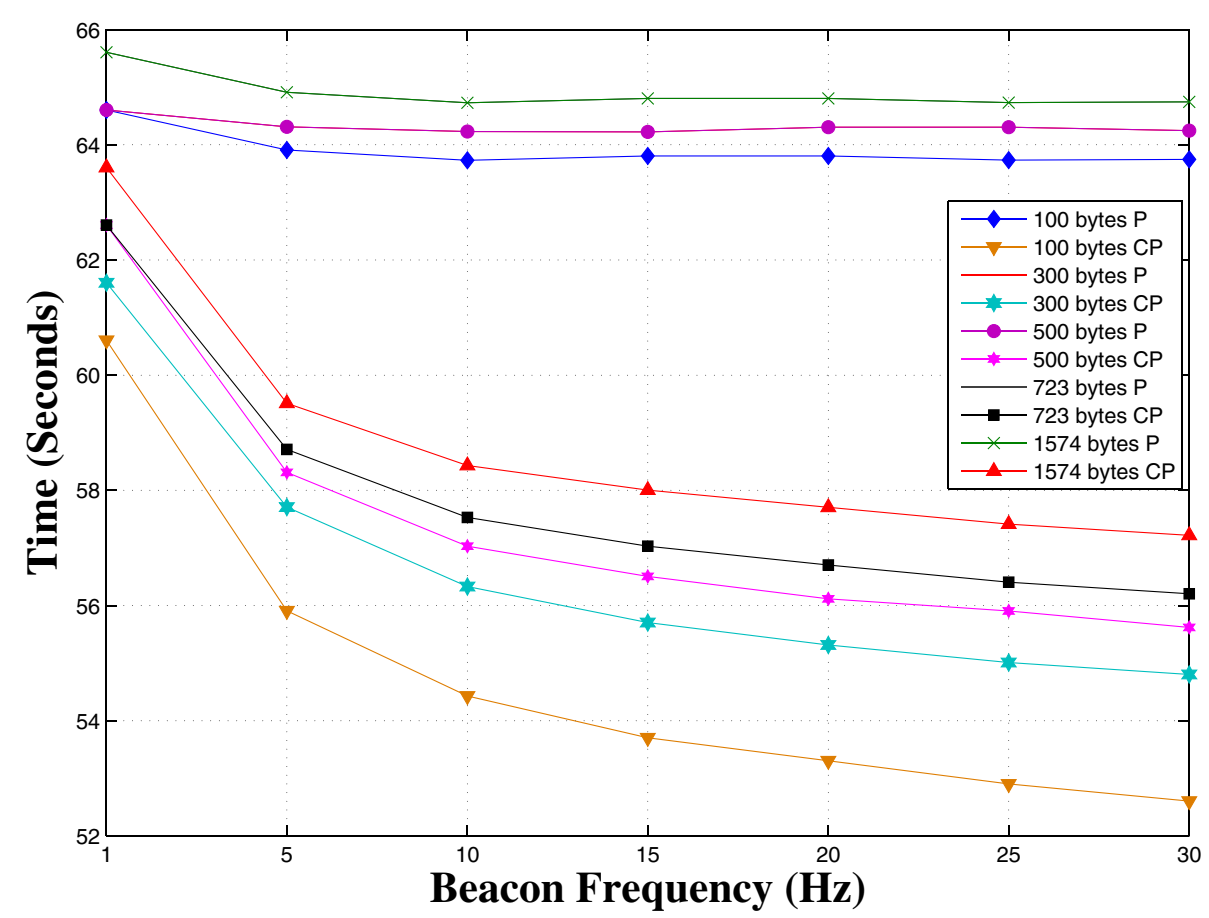

Figure $15 \mathrm{CP}$ reaching 1 (entry region $-30 \mathrm{~m} / \mathrm{s}$ ). 


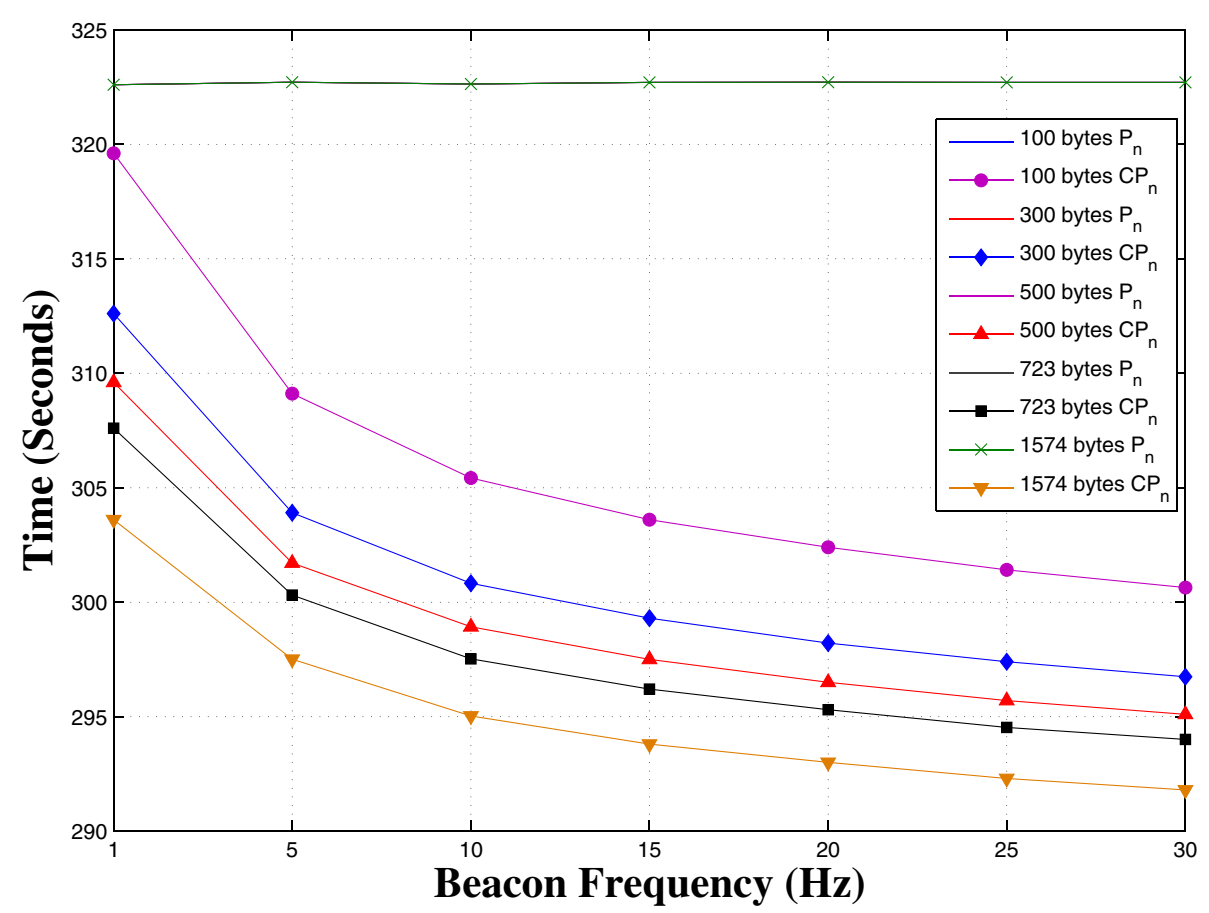

Figure $16 \mathrm{CP}_{n}$ reaching 0 (exit region $-10 \mathrm{~m} / \mathrm{s}$ ).

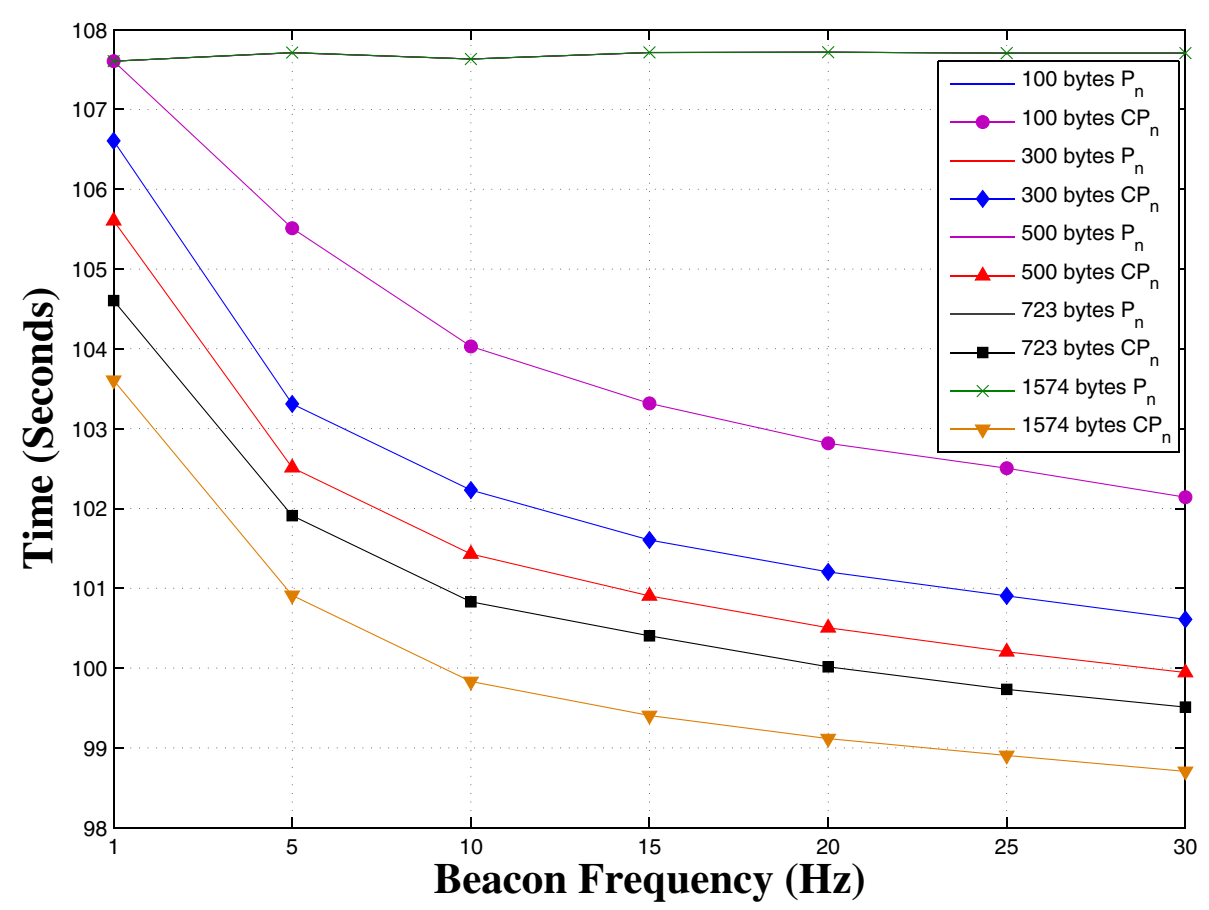

Figure $17 \mathrm{CP}_{n}$ reaching 0 (exit region $-30 \mathrm{~m} / \mathrm{s}$ ). 


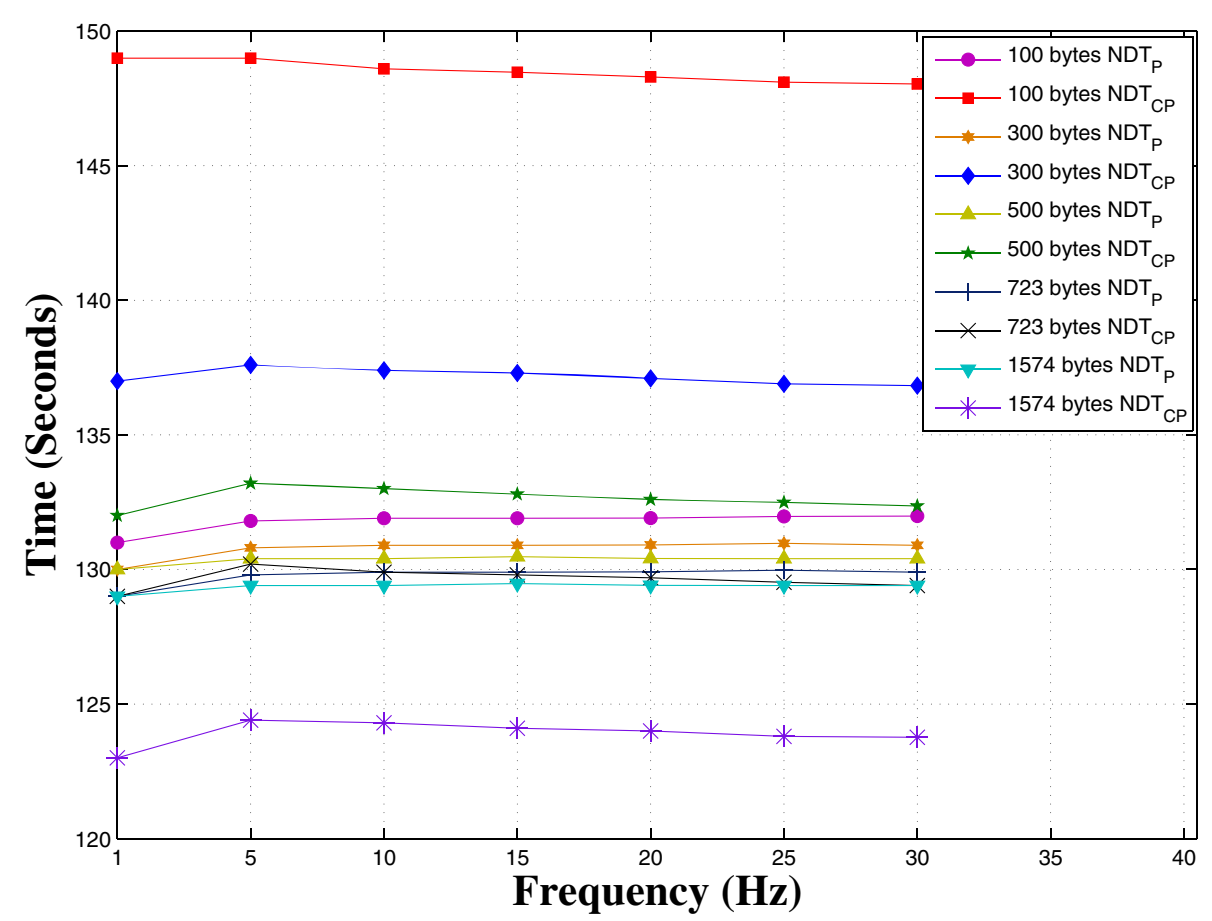

Figure 18 NDT of probability vs NDT of cumulative probability $(10 \mathrm{~m} / \mathrm{s})$.

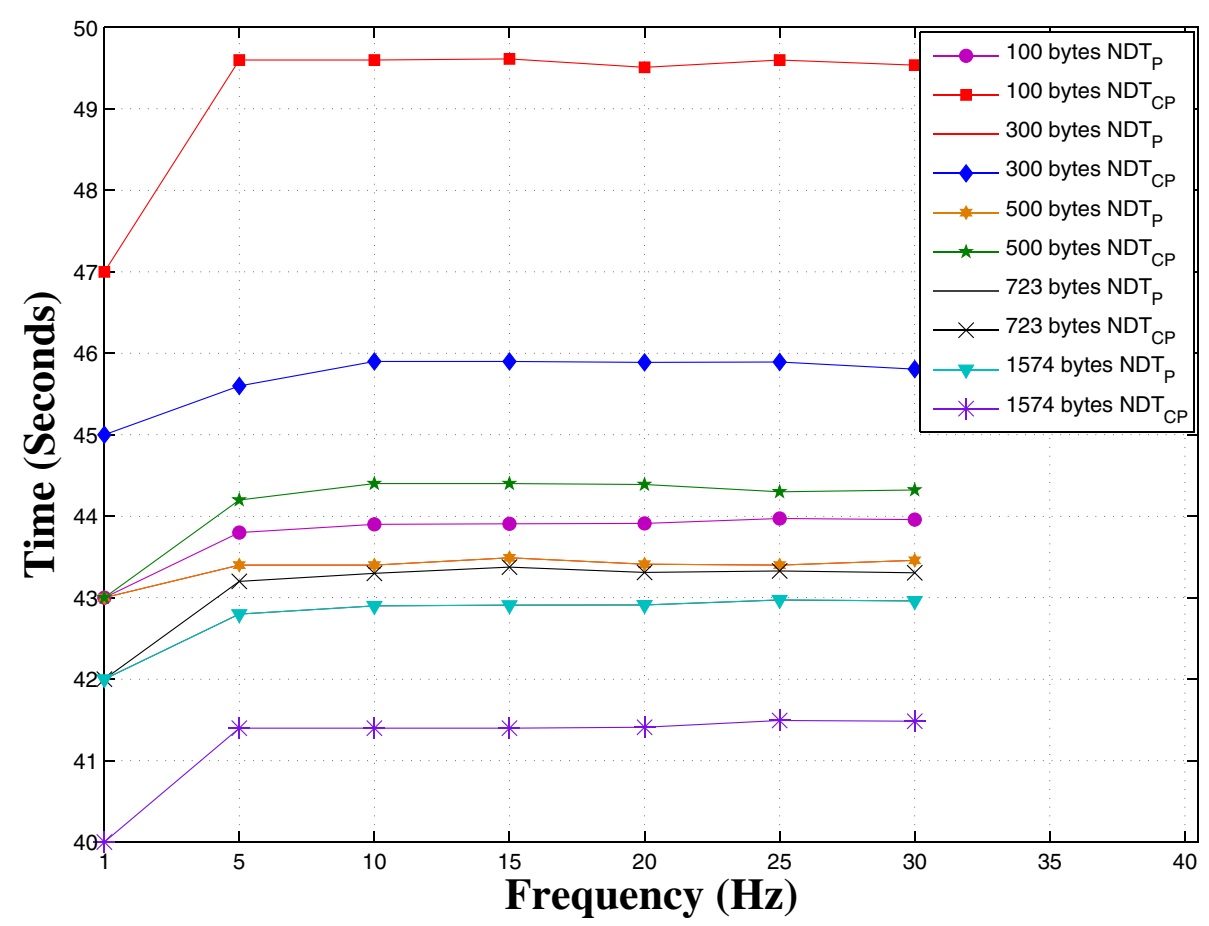

Figure 19 NDT of probability vs NDT of cumulative probability $(30 \mathrm{~m} / \mathrm{s})$. 


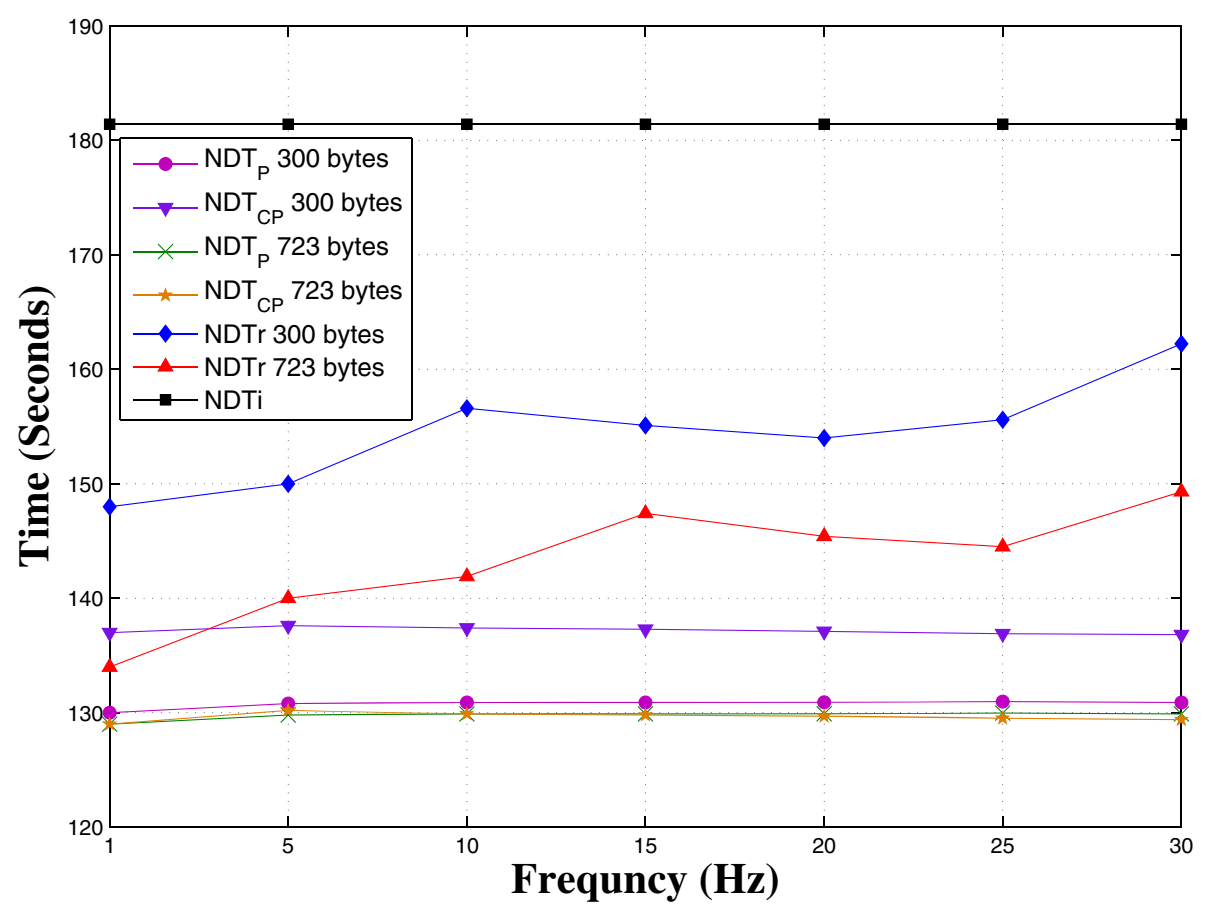

Figure 20 Comparison of P NDT vs CP NDT vs NDTr vs NDTi (10 m/s).

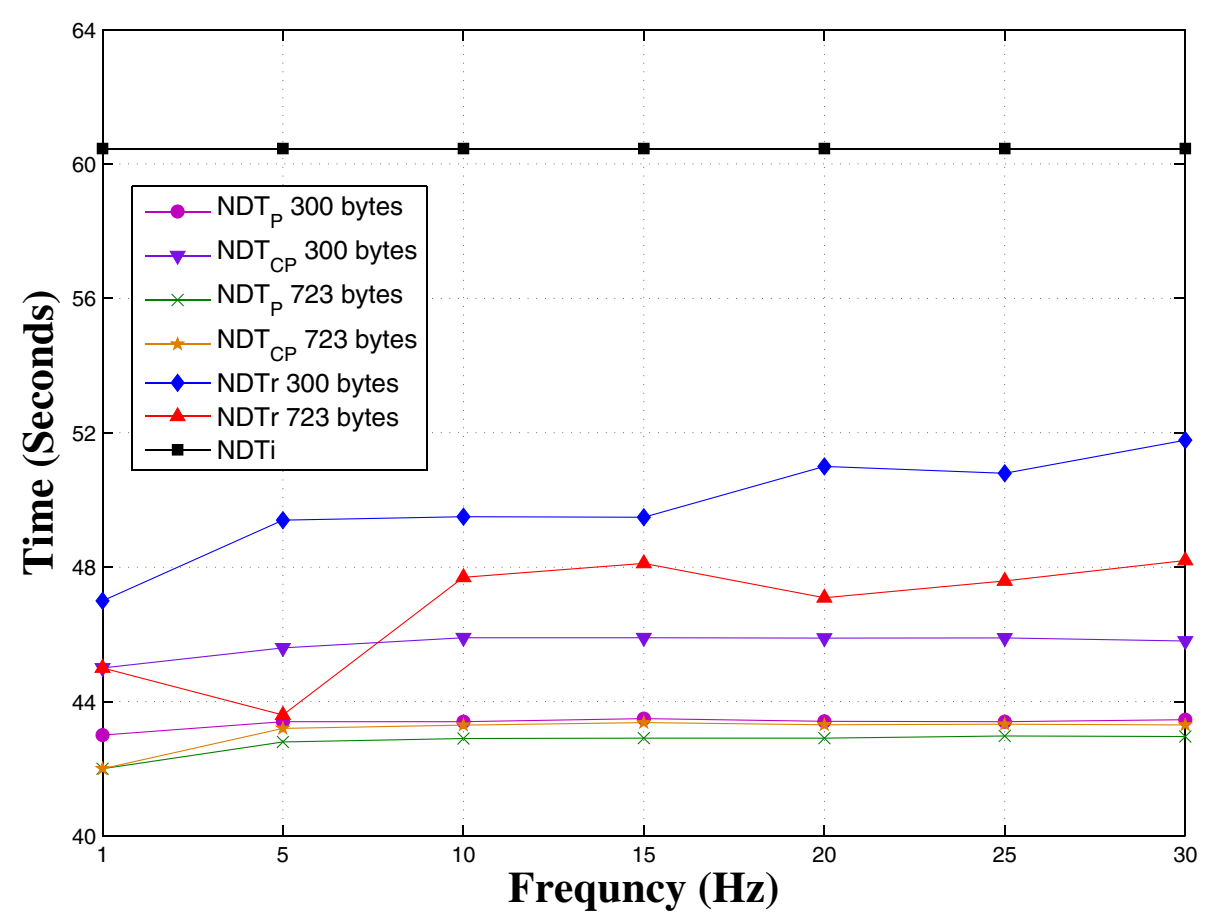

Figure 21 Comparison of P NDT vs CP NDT vs NDTr vs NDTi (30 m/s). 


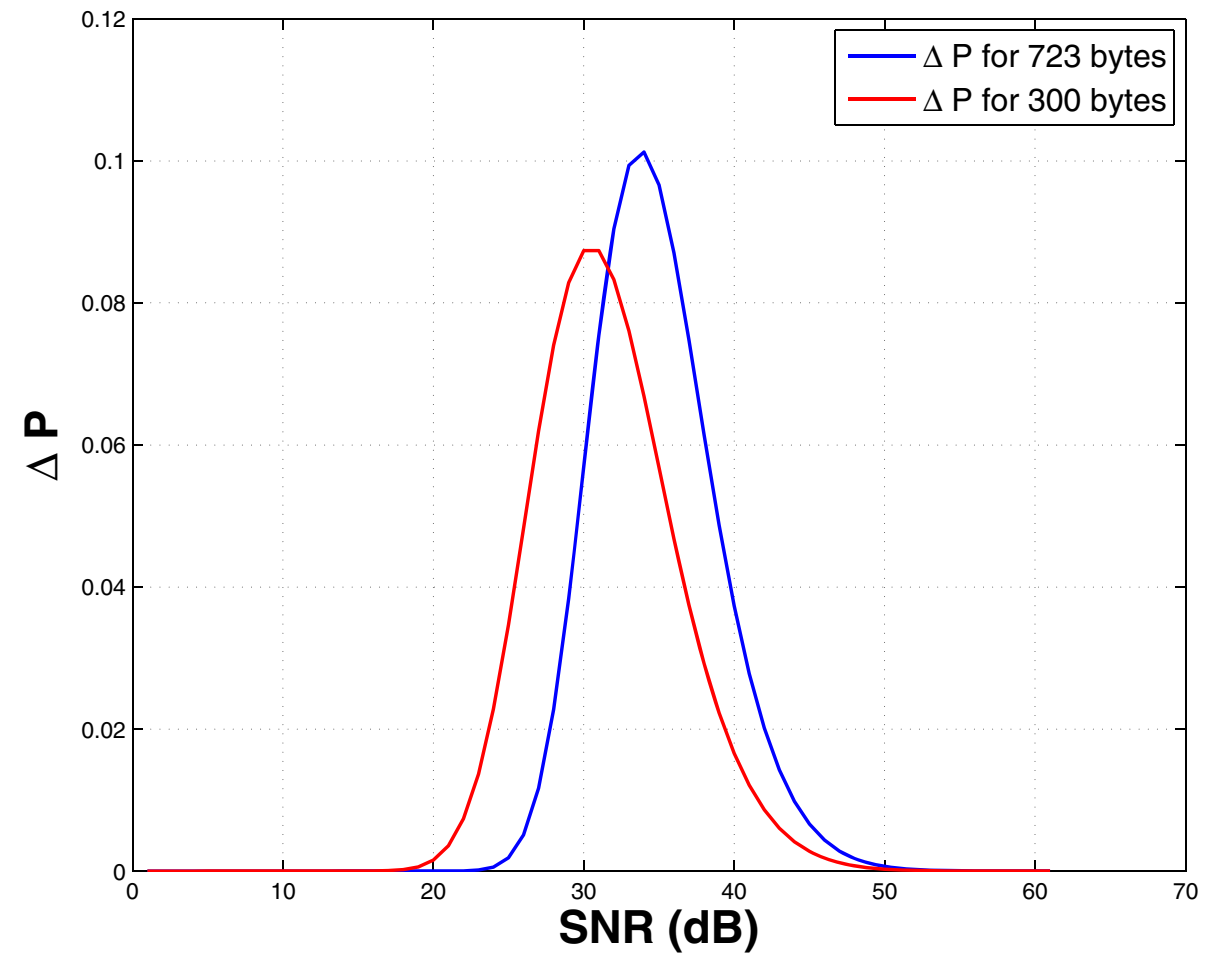

Figure $22 \Delta P$ vs SNR.

parameter that explains the relationship between beacon frequency and successful reception and not the individual probability.

The negative cumulative probability is the cumulative probability of no longer hearing (i.e. vehicle driving away from RSU) the beacon as the vehicle exits the coverage area. It could be thought of as the opposite of the positive cumulative probability when the vehicle enters the area. So the negative cumulative probability is the cumulative probability of $(1-P)$ where $P$ is the probability of successful packet reception. The graphs in Figures 16 and 17 show the negative cumulative probability and single packet reception probability for two velocities of vehicle.

The exit graph shown in Figure 17 also depicts exit times for different frequencies and different sizes so it clearly shows that the size of the packet affects the exit times due to fact that the probability of error and hence not hearing the packet increases with packet size and so the larger the packet size, the lower the exit times.

To obtain the NDT from our model, we subtract the exit times from the entry times of $\mathrm{CP}$ reaching ' 1 ' and this $\mathrm{NDT}$ is called as cumulative probability NDT $\left(N D T_{C P}\right)$ (i.e. NDT derived from CP). This means that NDT is being calculated based on the cumulative and single packet reception probabilities $\left(N D T_{P}\right)$ with the above results and depicted as a graphs in Figures 18 and 19.
The graphs in Figures 20 and 21 show NDTr, NDTi, $N D T_{P}$ and $N D T_{C P}$ for two different sizes of beacon. It is clear that these values are affected by the sizes of beacon. For relatively small beacon sizes, $N D T_{C P}$ is greater, but for much larger beacon sizes, the trend seems to be reversed. For beacon sizes around 723 bytes the $N D T_{C P}$ and $N D T_{P}$ are almost equal. This indicates that for handover, where predictability is important, maximum beacon sizes around 600 to 800 bytes (approx.) could give the best chance for seamless communication.

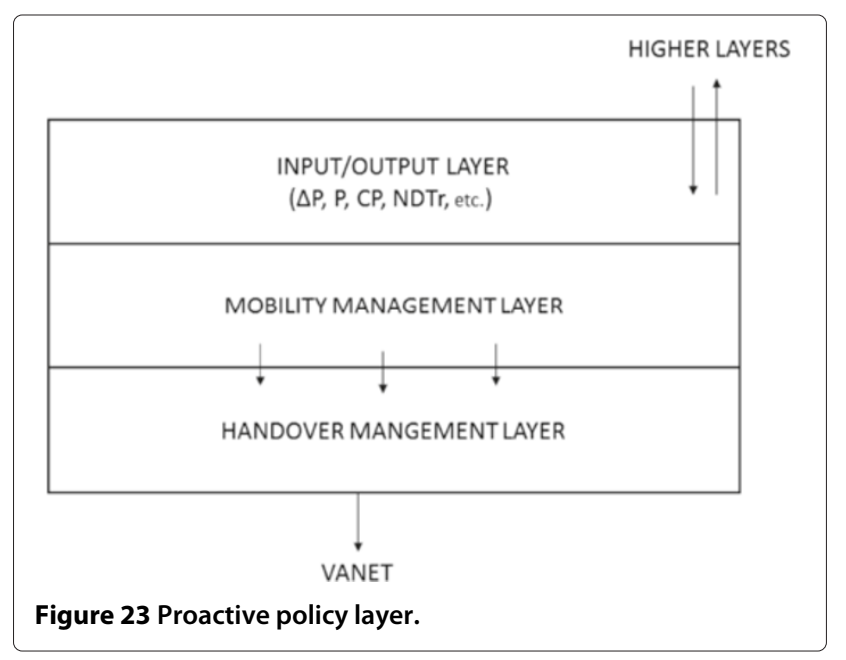




\subsection{The change in probability of successful beacon reception $(\Delta P)$ \\ 6.3.1 The change $(\Delta P)$ at entry}

For the entry region the rate of change in $P$, i.e. probability of successful beacon reception is shown in the Equation 11.

$$
\Delta P_{\text {ENTRY }}=P_{N}-P_{N-1}
$$

$\Delta P$ is significant because the SNR changes more rapidly with the increased velocity of the vehicle. Hence, $\Delta P$ increases significantly as the velocity of the vehicle increases. Where, $P_{N}$ is the probability of packet reception of an individual packet ' $\mathrm{N}$ ' and ' $\mathrm{N}-1$ ' is the previous packet. $\Delta P$ is calculated until $P$ reaches 1 .

\subsubsection{The change $(\Delta P)$ at exit}

For the exit region the rate of change in $P$ is as shown in the Equation 12.

$$
\Delta P_{\mathrm{EXIT}}=P_{N}-P_{N+1},
$$

where $P_{N}$ is the probability of packet reception of an individual packet ' $\mathrm{N}$ ' and ' $\mathrm{N}+1$ ' is the next packet. $\Delta P$ is calculated until $P$ reaches 0 .

The change in probability of successful beacon reception, i.e. $\Delta P$ vs SNR (dB) for beacon sizes 300 bytes and 723 bytes is illustrated as a graph in Figure 22. The graph is generated using the Equation 13 which do not take into account the velocity of vehicle. We know that $\Delta P$ for second packet (i.e $\mathrm{N}+1$ ) with respect to the first packet (i.e $N)$ can be calculated as

$$
\Rightarrow \Delta P=P_{2}-P_{1} .
$$

We know the formula for $P$, i.e.,

$$
\begin{aligned}
\Delta P= & {\left[1-1.5 \operatorname{erfc}\left(0.45 \sqrt{\mathrm{SNR}_{2}}\right)\right]^{L} } \\
& -\left[1-1.5 \operatorname{erfc}\left(0.45 \sqrt{\mathrm{SNR}_{1}}\right)\right]^{L}
\end{aligned}
$$

The simulation experiments were conducted to analyse the change in $P$ with respect to different velocities and different beacon frequencies. Due to vast amounts of results collected from the simulation, therefore, these results are available on request. These results clearly show the effect of size of beacon, velocity of vehicle and frequency of beacon. If a formula is being modelled based on these results, then for a given velocity of vehicle and for a given beacon size and frequency, the rate of change of $P$ can be calculated using the modelled formula. With this rate of change being known the $P$ and $\mathrm{CP}$ at any point can be calculated, which in turn can be used to predict the NDTr more accurately.

\section{Conclusions}

In this journal article, we have investigated the effect of beaconing on network dwell time using cumulative probability and individual successful beacon reception. This work has proved that the size of the beacon directly affects the individual packet reception probability (i.e. the value of $P$ changes), especially the probability distribution of the first packet P1 and hence affects both single reception probability as well as the cumulative reception probability. However, the frequency of the beacon only affects the cumulative probability, and hence, this shows that the effect of beacon size and beacon frequency is orthogonal to each other with regard to the network dwell time. In addition, the rate of change of the probability, i.e. $(\Delta P)$ is affected by the velocity of the vehicle and the velocity affects both the cumulative probability and the probability of successful reception. Hence, though the size and frequency of the beacon have orthogonal effects, the velocity of the vehicle affects both of these parameters. This work therefore significantly enhances our attempt to build a full-blown analytical model that encompasses all layers in an attempt to provide seamless handover.

\section{Future work}

Using the results of this journal article, we will develop a complete analytical framework which will be incorporated into the handover policy management mechanisms in mobile devices which will allow proactive seamless handover in both urban and motorway context as depicted in Figure 23. This will involve realistic techniques of estimating $\triangle P, P, C P$ and NDTr.

In the long term, we are seeking to develop a comprehensive framework that includes types of modulation being used as well as traffic density in order to handle seamless handover in both urban and motorway contexts.

\section{Competing interests}

The authors declare that they have no competing interests.

Received: 31 January 2014 Accepted: 3 November 2014 Published: 30 December 2014

\section{References}

1. G Karagiannis, O Altintas, E Ekici, G Heijenk, B Jarupan, K Lin, T Weil, Vehicular networking: a survey and tutorial on requirements, architectures, challenges, standards and solutions. Commun. Surv. Tutor IEEE. 13(4), 584-616 (2011). doi:10.1109/SURV.2011.061411.00019

2. A Ghosh, V Vardhan, G Mapp, O Gemikonakli, J Loo, in ITS Telecommunications (ITST) 2013 13th International Conference On. Providing ubiquitous communication using road-side units in VANET systems: unveiling the challenges, (2013), pp. 74-79. doi:10.1109/ ITST.2013.6685524

3. R Reinders, M van Eenennaam, G Karagiannis, G Heijenk, Contention Window Analysis for Beaconing in VANETs. Wireless Communications and Mobile Computing Conference (IWCMC), 2011, 7th International, 1481-1487 (2011). doi:10.1109/IWCMC.2011.5982757

4. E Van Eenennaam, G Karagiannis, G Heijenk, in Fourth ERCIM workshop on eMobility, Lule, Sweden. Towards Scalable Beaconing in VANETs, (2010), pp. 103-108. http://eprints.eemcs.utwente.nl/18015 
5. C Campolo, A Vinel, A Molinaro, Y Koucheryavy, Modeling Broadcasting in IEEE 802.11 p/WAVE Vehicular Networks. Commun. Lett. IEEE. 15(2), 199-201 (2011). doi:10.1109/LCOMM.2011.122810.102007

6. C Campolo, A Molinaro, A Vinel, in Vehicular Networking Conference (VNC) 2011 IEEE. Understanding the Performance of Short-Lived Control Broadcast packets in 802.11p/WAVE Vehicular Networks, (2011), pp. 102-108. doi:10.1109/NNC.2011.6117130

7. C Campolo, A Molinaro, A Vinel, Y Zhang, Modeling prioritized broadcasting in Multichannel Vehicular Networks. Vehicular Technol. IEEE Trans. 61(2), 687-701 (2012). doi:10.1109/TVT.2011.2181440

8. C Ganan, J Loo, A Ghosh, O Esparza, S Reñé, J Muñoz, in Multiple Access Communications. Lecture Notes in Computer Science, ed. by Bellalta B, Vinel A, Jonsson M, Barcelo J, Maslennikov R, Chatzimisios P, and Malone D. Analysis of inter-RSU Beaconing Interference in VANETs, vol. 7642 (Springer, 2012), pp. 49-59. doi:10.1007/978-3-642-34976-8_5

9. J-M Chung, M Kim, Y-S Park, M Choi, SW Lee, H-S Oh, Time Coordinated V2I Communications and Handover for WAVE Networks. Select. Areas Commun. IEEE J. 29(3), 545-558 (2011). doi:10.1109/JSAC.2011.110305

10. M van Eenennaam, WK Wolterink, G Karagiannis, G Heijenk, Exploring the Solution Space of Beaconing in VANETs. Vehicular Networking Conference (VNC), 2009, IEEE, 1-8 (2009). doi:10.1109/VNC.2009.5416370

11. GE Mapp, F Katsriku, M Aiash, N Chinnam, R Lopes, E dos Santos Moreira, RMP Vanni, M Augusto, Exploiting ocation and contextual information to develop a comprehensive framework for Proactive Handover in Heterogeneous Environments. J. Comput. Netw. Commun, 1-17 (2012). doi:10.1155/2012/748163

12. F Shaikh, G Mapp, A Lasebae, in Next Generation Mobile Applications, Services and Technologies, 2007. NGMAST'07. The 2007 International Conference On. Proactive Policy Management using TBVH mechanism in Heterogeneous Networks, (2007), pp. 151-157. doi:10.1109/NGMAST. 2007.4343414

13. A Varga, OMNeT++: an extensible, modular, component-based C++ Network Simulation. http://www.omnetpp.org/

14. J-P Sheu, C-Y Lo, W-K Hu, in Parallel and Distributed Systems (ICPADS) 2011 IEEE 17th International Conference On. A Distributed Routing Protocol and Handover Schemes in Hybrid Vehicular ad hoc Networks, (2011), pp. 428-435. doi:10.1109/ICPADS.2011.4

15. J Dias, A Cardote, F Neves, S Sargento, A Oliveira, Seamless Horizontal and Vertical Mobility in VANET. Vehicular Networking Conference (VNC), 2012, IEEE, 226-233 (2012). doi:10.1109/VNC.2012.6407436

16. J Gozalvez, M Sepulcre, R Bauza, IEEE 802.11 p vehicle to Infrastructure Communications in Urban Environments. Commun. Mag. IEEE. 50(5), 176-183 (2012). doi:10.1109/MCOM.2012.6194400

17. A Bohm, M Jonsson, Handover in IEEE 802.11p-based Delay-Sensitive Vehicle-to-Infrastructure Communication, (Technical Report IDE - 0924, Halmstad University, Embedded Systems (CERES), 2009)

18. C-C Tseng, K-H Chi, M-D Hsieh, H-H Chang, Location-Based Fast Handoff for 802.11 Networks. Commun. Lett. IEEE. 9(4), 304-306 (2005). doi:10.1109/LCOMM.2005.04010

19. J Montavont, T Noel, in Wireless and Mobile Computing, Networking and Communications, 2006. (WiMob'2006). IEEE International Conference On. IEEE 802.11 Handovers assisted by GPS Information, (2006), pp. 166-172. doi:10.1109/WIMOB.2006.1696358

20. EK Paik, Y Choi, in Telecommunications, 2003. ICT 2003. 10th International Conference On. Prediction-based fast handoff for Mobile WLANs, vol. 1, (2003), pp. 748-7531. doi:10.1109/ICTEL.2003.1191503

21. H Lee, Y-U Chung, Y-H Choi, in Ubiquitous and Future Networks (ICUFN) 2013 Fifth International Conference On. A seamless Handover Scheme for IEEE WAVE Networks based on multi-way Proactive Caching, (2013), pp. 356-361. doi:10.1109/ICUFN.2013.6614841

22. ME Augusto, R Porto, Guardia Vanni HC, M Aiash, G Mapp, E dos Santos Moreira, MYHand, A Novel Architecture for Improving Handovers in NGNs. http://www.thinkmind.org/index.php?view=article\&articleid= aict_2013_9_40_10181

23. IEEE-Std, IEEE Draft Standard for Local and metropolitan area networks Part 21: Media Independent Handover Services - Amendment 3: Optimized Single Radio Handovers, IEEE P802.21c/D6, August 2013, Dec. 25 2013, (2013), pp. 1-70. http://ieeexplore.ieee.org/stamp/stamp.jsp?tp=\& arnumber $=6634309 \&$ isnumber $=6634308$

24. GE Mapp, F Shaikh, D Cottingham, J Crowcroft, J Baliosian, in Proceedings of the 3rd International Conference on Wireless Internet. Y-comm: A Global
Architecture for Heterogeneous Networking (WICON '07, (ICST Institute for Computer Sciences, Social-Informatics and Telecommunications Engineering) ICST, Brussels, Belgium, Belgium, 2007), pp. 22-1225. http:// dl.acm.org/citation.cfm?id=1460047.1460075

25. T Salam, M Ali, M-R Fida, in Information Technology: New Generations (ITNG) 2011 Eighth International Conference On. Seamless Proactive Vertical Handover Algorithm, (2011), pp. 94-99. doi:10.1109/ITNG.2011.24

26. C Sommer, VEINS: Vehicles In Network Simulation. http://veins.car2x.org/

27. C Sommer, R German, F Dressler, Bidirectionally Coupled Network and Road Traffic Simulation for Improved IVC Analysis. Mobile Comput. IEEE Trans. 10(1), 3-15 (2011). doi:10.1109/TMC.2010.133

28. IEEE-Std., IEEE Draft Standard for Wireless Access in Vehicular Environments (WAVE) - Multi-Channel Operation, IEEE 1609.4/D8.0, June 2010, 1-92 (July 15 2010). http://ieeexplore.ieee.org/stamp/stamp.jsp? $\mathrm{tp}=$ \&arnumber $=5511464$ \&isnumber $=5511463$

29. IEEE Standard for Information technology-local and metropolitan area networks-specific requirements-Part 11: wireless LAN medium access control (MAC) and physical layer (PHY) specifications - amendment 8: medium access control (MAC) quality of service enhancements. doi:10.1109/IEEESTD.2005.97890

30. K Sjoberg, J Kaa redal, M Moe, O Kristiansen, R Soraa sen, E Uhlemann, F Tufvesson, K Evensen, E Stro m, in 17th World Congress on Intelligent Transport Systems. Measuring and Using the RSSI of IEEE 802.11p, vol. 2010 (Busan, 2010), pp. 1-9. http://lup.lub.lu.se/luur/download?func= downloadFile\&recordOld=2607975\&fileOld =2607991

31. P Fuxjager, A Costantini, D Valerio, P Castiglione, G Zacheo, T Zemen, F Ricciato, in 6th Karlsruhe Workshop on Software Radios (WSR). IEEE 802.11p Transmission Using GNURadio (Karlsruhe, Germany, 2010), pp. 1-4. http:// www-int.etec.uni-karlsruhe.de/seiten/conferences/past/WSR2010/ Papers/wsr10_13.pdf

doi:10.1186/1687-1499-2014-227

Cite this article as: Ghosh et al:: Exploring efficient seamless handover in VANET systems using network dwell time. EURASIP Journal on Wireless Communications and Networking 2014 2014:227.

\section{Submit your manuscript to a SpringerOpen ${ }^{\circ}$ journal and benefit from:}

- Convenient online submission

Rigorous peer review

- Immediate publication on acceptance

- Open access: articles freely available online

- High visibility within the field

- Retaining the copyright to your article

Submit your next manuscript at $>$ springeropen.com 